\title{
On the Stokes Equation with the Leak and Slip Boundary Conditions of Friction Type: Regularity of Solutions
}

By

\author{
Norikazu SAITO*
}

\begin{abstract}
We consider the Stokes equations under some nonlinear boundary conditions, which are described in terms of subdifferentials of maximal monotone graphs and are called leak and slip boundary conditions of friction type. The main objective is to show the existence of strong solutions, say $u \in H^{2}$ and $p \in H^{1}$, to these problems. We start with weak solutions to variational inequalities, and then study the regularity of weak solutions. Our main theorems imply the maximality of Stokes operators with such nonlinear boundary conditions in a suitable Hilbert space and they are of use in analysis of time-dependent problems. Linear boundary conditions of Neumann type, such as slip and penetration conditions, are also discussed.
\end{abstract}

\section{$\S 1 . \quad$ Introduction}

Let $\Omega$ be a bounded domain in $\mathbb{R}^{N}, N=2,3$. We suppose that the boundary $\partial \Omega$ of $\Omega$ is composed of two connected components $\Gamma$ and $\Gamma_{D}$ which are assumed to be Lipschitz continuous, unless otherwise stated. $\Gamma$ is not empty, whereas $\Gamma_{D}$ may be empty. In the present paper, we shall mainly discuss the existence of a strong solution $u \in H^{2}(\Omega)^{N}$ and $p \in H^{1}(\Omega)$ to a modified Stokes equation

$$
\lambda u-\Delta u+\nabla p=f, \quad \operatorname{div} u=0 \quad \text { in } \Omega
$$

Communicated by H. Okamoto. Received November 20, 2002. Revised May 22, 2003. 2000 Mathematics Subject Classification(s): 35B65, 35J85, 35Q30

Key words: regularity, variational inequality, Stokes equation, nonlinear boundary condition

Supported by Grants-in-Aid for Scientific Research (No. 14740067), The Japan Society of the Promotion of Science (JSPS).

*Faculty of Education, Toyama University, Gofuku 3190, Toyama 930-8555 Japan.

e-mail: saito@edu.toyama-u.ac.jp 
under the standard Dirichlet boundary condition

$$
u=0 \quad \text { on } \Gamma_{D}
$$

together with the one of the following nonlinear boundary conditions:

$$
\begin{array}{ll}
u_{\tau}=0, \quad-\sigma_{n} \in g \partial\left|u_{n}\right| & \text { on } \Gamma, \\
u_{n}=0, \quad-\sigma_{\tau} \in g \partial\left|u_{\tau}\right| & \text { on } \Gamma .
\end{array}
$$

Here, $\lambda$ denotes a non-negative constant; $u=\left(u_{1}, \ldots, u_{N}\right)$ the velocity vector and $p$ the pressure; $f$ and $g(\geq 0)$ given vector and scalar functions; $u_{n} \equiv u \cdot n$ and $u_{\tau} \equiv u-n u_{n}$ are the normal and tangential components of the velocity, respectively, where $n=\left(n_{1}, \ldots, n_{N}\right)$ stands for the outer unit normal to $\Gamma$; $\sigma_{n}=\sigma_{n}(u, p)$ and $\sigma_{\tau}=\sigma_{\tau}(u)$ denote normal and tangential components of the stress vector (the precise definitions will be recalled in $\S 2$ ); and finally $\partial|z|$ denotes a graph

$$
\partial|z|= \begin{cases}\frac{z}{|z|} & \left(z \neq 0, z \in \mathbb{R}^{m}\right) \\ \left\{w \in \mathbb{R}^{m}|| w \mid \leq 1\right\} & \left(z=0, z \in \mathbb{R}^{m}\right)\end{cases}
$$

where $m=1$ for (1.3) and $m=N$ for (1.4). It is easy to see that the second conditions of (1.3) and (1.4) are equivalent to

$$
\begin{array}{lll}
\left|\sigma_{n}\right| \leq g, & \sigma_{n} u_{n}+g\left|u_{n}\right|=0 & \text { on } \Gamma \\
\left|\sigma_{\tau}\right| \leq g, & \sigma_{\tau} \cdot u_{\tau}+g\left|u_{\tau}\right|=0 & \text { on } \Gamma
\end{array}
$$

respectively.

The problem composed of (1.1), (1.2) and (1.3), which we will refer as the leak boundary problem of friction type, or simply (Pr. LF), was introduced by H. Fujita ([9]) in order to study steady motions of viscous incompressible fluid involving a leak of the fluid through the surface or penetration into adjacent media. Applications to oil flow over or beneath sand layers are presented in Kawarada, Fujita and Suito [19], and Kawarada and Suito [20].

In [9], the existence and uniqueness/non-uniqueness of a weak solution, say $u \in H^{1}(\Omega)^{N}$ and $p \in L^{2}(\Omega)$, has been established by means of a variational inequality and an extended Hanh-Banach theorem. We shall review his results in $\S 3$.

The first purpose of the present paper is to study the regularity of Fujita's weak solution, and prove the following theorem. 
Theorem 1.1. Let $\lambda \geq 0$. Suppose that the following assumptions hold:

$$
\begin{aligned}
& \bar{\Gamma} \cap \overline{\Gamma_{D}}=\emptyset ; \\
& \Gamma_{D} \text { and } \Gamma \text { are of class } C^{2} \text { and } C^{4} \text {, respectively; } \\
& \Gamma_{D} \neq \emptyset \quad(\text { if } \lambda=0) ; \\
& g \in H^{1 / 2}(\Gamma), \quad g \geq 0 \text { a.e.in } \Gamma ; \\
& f \in L^{2}(\Omega)^{N} .
\end{aligned}
$$

Then there exists a solution $\{u, p\} \in H^{2}(\Omega)^{N} \times H^{1}(\Omega)$ of

(Pr. LF)

$$
\begin{cases}\lambda u-\Delta u+\nabla p=f, \quad \operatorname{div} u=0 & \text { in } \Omega, \\ u=0 & \text { on } \Gamma_{D}, \\ u_{\tau}=0, \quad-\sigma_{n} \in g \partial\left|u_{n}\right| & \text { on } \Gamma .\end{cases}
$$

The velocity $u$ is unique, while the pressure $p$ is unique except for an additive constant. The range of the additive constant to $p$ is limited to $\{0\}$ or to a finite closed interval. Furthermore there is a positive constant depending only on $\Omega$ such that

$$
\begin{aligned}
& \|u\|_{H^{2}(\Omega)^{N}}+\|p\|_{H^{1}(\Omega)} \\
& \leq C\left(\|f\|_{L^{2}(\Omega)^{N}}+\|g\|_{H^{1 / 2}(\Gamma)}+\|u\|_{H^{1}(\Omega)^{N}}+\|p\|_{L^{2}(\Omega)}\right)
\end{aligned}
$$

for any solution $\{u, p\}$ of (Pr. LF).

Several remarks are in order.

Remark. The description about non-uniqueness of the pressure is rather troublesome. Let $\{u, p\}$ solve (Pr. LF). For the sake of simplicity, we assume that $\sigma_{n}(u, p)$ and $g$ are continuous. Following [9], we set

$$
k_{1}=\sup _{\Gamma}\left(\sigma_{n}(u, p)-g\right), \quad k_{2}=\inf _{\Gamma}\left(\sigma_{n}(u, p)+g\right) .
$$

Obviously $-2\|g\|_{L^{\infty}(\Gamma)} \leq k_{1} \leq k_{2} \leq 2\|g\|_{L^{\infty}(\Gamma)}$. Then a function $p+k, k \in$ $\left[k_{1}, k_{2}\right]$, is also a corresponding pressure of $u ;\{u, p+k\}$ also solve (Pr. LF). If another corresponding pressure $p^{*}$ has been taken first, then the value of $k_{1}, k_{2}$ are changed. On the other hand, if non-trivial movement $\left(u_{n} \neq 0\right)$ takes place on a portion $\Gamma_{1} \subset \Gamma$, then $p$ is uniquely determined. This means that the range of the additive constant to $p$ is limited to $\{0\}$. See, for more detail, Remark 3.2 of $[9]$. 
We here give a simple example to illustrate this issue. We employ the polar coordinates $x=(r, \theta)$ in $\mathbb{R}^{2}$. We assume that

$$
\Omega=\{(r, \theta) \mid 1<r<2\}, \quad \Gamma_{D}=\{r=1\}, \quad \Gamma=\{r=2\},
$$

and set $e_{r}=(\cos \theta, \sin \theta), e_{\theta}=(-\sin \theta, \cos \theta)$. Put $u(r, \theta)=w(r) e_{\theta}$ and $p(r)=\kappa r$, where $w(r)=4 r^{-1}+2 r-6$ and $\kappa>0$ is a constant. Observe that $\{u, p\}$ solves $-\Delta u+\nabla p=-6 e_{\theta} r^{-2}+\kappa e_{r}, \operatorname{div} u=0$ in $\Omega,\left.u\right|_{\Gamma_{D}}=0,\left.u_{\tau}\right|_{\Gamma}=0$, and $\left.u_{n}\right|_{\Gamma}=0$. We have $\sigma_{n}(u, p)=-p(r)$ and hence $\left|\sigma_{n}(u, p)\right|=2 \kappa$. We define $g=2 \kappa+1$. Then $\left|\sigma_{n}(u, p)\right|<g$ and $u_{\tau}, u_{n}$ vanish on $\Gamma$. Therefore $\{u, p\}$ is a solution of (Pr. LF). In this case, we have $k_{1}=-1-4 \kappa$ and $k_{2}=1$. Now put $p_{k}=p+k$ with a constant $k$. As long as $k$ is taken from $\left[k_{1}, k_{2}\right],\left\{u, p_{k}\right\}$ solves (Pr. LF). However, if $k \notin\left[k_{1}, k_{2}\right]$, then $\left\{u, p_{k}\right\}$ does not solve (Pr. LF), since $\left|\sigma_{n}\left(u, p_{k}\right)\right|>g$.

Remark. Assumption that $g \in H^{1 / 2}(\Gamma)$ is really optimal to derive the $H^{2}-H^{1}$ regularity of $\{u, p\}$. We shall revisit this issue in the paragraph (D) of $\S 7$, after having prepared a few materials to discuss it.

Remark. Theorem 1.1 has an important application on evolution problems. Actually, Theorem 1.1 implies the maximality of the operator $A$ on $X=L^{2}(\Omega)^{N}$ defined as

$$
A u=\{-\Delta u+\nabla p \mid p \in M(u)\} \quad(u \in D(A))
$$

with

$$
D(A)=\left\{u \in H^{2}(\Omega)^{N}|\operatorname{div} u=0, u|_{\Gamma_{D}}=0,\left.u_{\tau}\right|_{\Gamma}=0, M(u) \neq \emptyset\right\},
$$

where

$$
M(u)=\left\{p \in H^{1}(\Omega)\left|-\sigma_{n} \in g \partial\right| u_{n} \mid \text { on } \Gamma\right\} .
$$

That is, we have $u \in D(A)$ satisfying $\lambda u+A u=f$ in $X$ for all $f \in X$ and $\lambda>0$. On the other hand, it is easy to verify that $A$ is monotone in $X$; $\left(f_{1}-f_{2}, u_{1}-u_{2}\right) \geq 0$ for all $u_{1}, u_{2} \in D(A)$ and $f_{1} \in A u_{1}, f_{2} \in A u_{2}$. These enable us to apply the nonlinear semigroup theory in $X$ ([21]) and lead to the unique solvability of a non-stationary Stokes problem

$$
\frac{\partial u}{\partial t}=\Delta u-\nabla p, \quad \operatorname{div} u=0
$$


with boundary conditions (1.2), (1.3) and the initial condition $\left.u\right|_{t=0}=u_{0}(x)$. For more detail, see Fujita [10], [11] and [31]. Moreover, by developing Fujita's argument with the aid of monotonicity method ([3], [5]), under a suitable assumption on $f=f(x, t)$, we can solve

$$
\frac{\partial u}{\partial t}=\Delta u-\nabla p+f, \quad \operatorname{div} u=0
$$

with (1.2), (1.3), $\left.u\right|_{t=0}=u_{0}(x)$. We shall report the detail and an application to the non-stationary Navier-Stokes equations in a forthcoming paper.

The second purpose of this paper is to study the slip boundary value problem of friction type, (Pr. SF), which is composed of (1.1), (1.2) and (1.4). This problem appears in modelling of blood flow in a vein of an arterial sclerosis patient and in that of avalanche of water and rocks ([9]). Concerning (Pr. SF), we have the following theorem where we should keep in mind that $\sigma_{\tau}$ does not explicitly contain $p$.

Theorem 1.2. Let $\lambda \geq 0$, and suppose (1.6), (1.7), (1.8), (1.9), and (1.10). Then there exists a unique solution $\{u, p\} \in H^{2}(\Omega)^{N} \times H^{1}(\Omega)$ of

$$
\begin{cases}\lambda u-\Delta u+\nabla p=f, \quad \operatorname{div} u=0 & \text { in } \Omega \\ u=0 & \text { on } \Gamma_{D} \\ u_{n}=0, \quad-\sigma_{\tau} \in g \partial\left|u_{\tau}\right| & \text { on } \Gamma\end{cases}
$$

satisfying

$$
\int_{\Omega} p d x=0 .
$$

Moreover we have

$$
\begin{aligned}
& \|u\|_{H^{2}(\Omega)^{N}}+\|p\|_{H^{1}(\Omega)} \\
& \quad \leq C\left(\|f\|_{L^{2}(\Omega)^{N}}+\|g\|_{H^{1 / 2}(\Gamma)}+\|u\|_{H^{1}(\Omega)^{N}}+\|p\|_{L^{2}(\Omega)}\right) .
\end{aligned}
$$

In $\S 2$, we shall introduce the notation used in this paper and describe some identities and lemmas. In order to prove Theorem 1.1, we begin by considering a weak formulation (Pr. WLF) to (Pr. LF) by means of a variational inequality which was proposed by [9]. However, following Brezis [4], we do not treat (Pr. WLF) directly and study a regularized problem (Pr. $\left.\mathrm{WLF}_{\varepsilon}\right)$ to $(\mathrm{Pr}$. 
WLF), where $\varepsilon>0$ denotes a regularized parameter. Actually, $\left(\operatorname{Pr} . \mathrm{WLF}_{\varepsilon}\right)$ is defined via the Yosida regularization of $\partial|\cdot|$. Taking such detour allows us to avoid a redundant hypothesis on $g$. On the other hand, from the viewpoint of numerical analysis, a regularized problem itself is worth considering. We shall describe $(\operatorname{Pr} . \mathrm{WLF})$ and $\left(\operatorname{Pr} . \mathrm{WLF}_{\varepsilon}\right)$ in $\S 3$. The unique existence of a solution to $\left(\operatorname{Pr} . \mathrm{WLF}_{\varepsilon}\right)$ is also mentioned there. In $\S 4$, we investigate the regularity of a solution to $\left(\operatorname{Pr} . \mathrm{WLF}_{\varepsilon}\right)$ and then establish the proof of Theorem 1.1. The strategy is standard in regularization theory. That is, we employ a localization technique, partition of unity and difference quotients. The method of the proof of Theorem 1.2 is given in $\S 5$.

In $\S 6$, although digressing from the main subject, we consider a few kinds of linear boundary value problems of Neumann type for the Stokes equations. Specifically, we shall deal with (1.1) and (1.2) under one of the following conditions to be posed on $\Gamma$ :

$$
\begin{array}{lll}
u_{\tau}=0, \quad \sigma_{n}=\omega_{n} & \text { (non-slip and penetration); } \\
u_{n}=0, \quad \sigma_{\tau}=\omega_{\tau} & \text { (non-penetration and slip); } \\
\sigma=\omega & & \text { (prescribed stress) }
\end{array}
$$

where $\omega_{n}, \omega_{\tau}$ and $\omega$ are given functions defined on $\Gamma$. Here, for example, the condition (1.12) means that the fluid under consideration does not slip at the boundary and the penetration of the fluid through the boundary is controlled by a prescribed "force of stream" which is expressed as the normal component of the stress. We are concerned with the regularity of weak solutions to these problems such as $u \in H^{k+2}$ and $p \in H^{k+1}, k \geq 0$ (Theorems 6.1, 6.2, and 6.3 described below). To accomplish these, in addition to the argument of $\S 4$, following Bello [2], we adopt a well-known theorem on abstract variational problems with constraints, which gives a better viewpoint in the proof.

As is well-known, Neumann boundary value problems described above play a fundamental role in analysis of some actual and practical problems including free boundary problems ([24], [27], [28]) and a technique of numerical methods ([18]). However, concerning regularity results of weak solutions, we could find no explicit reference to them in the literature. In fact, (1.1), (1.2) with the condition (1.12) in the case of $\omega_{n} \equiv 0$ was described in Solonnikov and Šcadilov [28]. But it seems that the complete proof for the case of $\omega_{n} \not \equiv 0$ is not explicitly stated there. This is why we decide to state an explicit and a somewhat elementally proof in this paper, although they seem not to be new for specialists. 
Finally, in $\S 7$, we mention several additional remarks including a commentary to the full stress problem $([26])$ and a relation with a general theory of Agmon, Douglis and Nirenberg [1].

\section{$\S 2 . \quad$ Preliminaries}

(A) Notation. The deformation tensor and stress tensor associated with a velocity field $u=\left(u_{1}, \ldots, u_{N}\right)$ and pressure $p$ are denoted by

$$
\left[e_{i j}(u)\right] \quad e_{i j}(u)=\frac{\partial u_{i}}{\partial x_{j}}+\frac{\partial u_{j}}{\partial x_{i}} \quad \text { and } \quad\left[S_{i j}(u, p)\right] \quad S_{i j}(u)=-p \delta_{i j}+e_{i j}(u),
$$

respectively, where $\delta_{i j}$ denotes Kroneker's delta. The stress vector $\sigma(u, p)$ is defined by $\sigma(u, p)=\left[S_{i j}(u, p)\right] n$ of which the $i$ th component is

$$
\sum_{j=1}^{N} S_{i j}(u, p) n_{j}
$$

The normal and tangential components of a vector field $u$ are defined as $u_{n}=$ $u \cdot n$ and $u_{\tau}=u-n u_{n}$, respectively. In particular,

$\sigma_{n}(u, p)=\sigma(u, p) \cdot n \quad$ (the normal component of the stress vector);

$\sigma_{\tau}(u)=\sigma(u, p)-n \sigma_{n}(u, p) \quad$ (the tangential component of the stress vector).

If there is no possibility of confusion, we simply write $\sigma, \sigma_{n}$ and $\sigma_{\tau}$ to express $\sigma(u, p), \sigma_{n}(u, p)$ and $\sigma_{\tau}(u)$, respectively.

We will use the $L^{2}(\Omega)$ space and the usual Sobolev spaces $H^{m}(\Omega)$ for a non-negative integer $m . H^{0}(\Omega)$ is understood as $L^{2}(\Omega)$. We put

$$
L_{0}^{2}(\Omega)=\left\{v \in L^{2}(\Omega) \mid \int_{\Omega} v d x=0\right\}
$$

We write $\|\cdot\|_{m}=\|\cdot\|_{m, \Omega}$ instead of $\|\cdot\|_{H^{m}(\Omega)}$, and set $\|\cdot\|=\|\cdot\|_{0}$.

We also use the Sobolev space $H^{s}(\Gamma)$ defined on the boundary $\Gamma$, where $s \in \mathbb{R}$. We write $\|\cdot\|_{s, \Gamma}=\|\cdot\|_{H^{s}(\Gamma)} \cdot H^{0}(\Gamma)$ is understood as $L^{2}(\Gamma)$. The surface element of $\Gamma$ is denoted by $d s$, that is

$$
\|\eta\|_{0, \Gamma}^{2}=\int_{\Gamma}|\eta|^{2} d s
$$

We write $(\cdot, \cdot)=(\cdot, \cdot)_{L^{2}(\Omega)}$ and $(\cdot, \cdot)_{\Gamma}=(\cdot, \cdot)_{L^{2}(\Gamma)}$. 
Let $\operatorname{Tr}$ be the trace operator from $H^{1}(\Omega)$ into $H^{1 / 2}(\Gamma)$. Then the trace $\operatorname{Tr} v$ on $\Gamma$ of $v \in H^{1}(\Omega)$ is denoted by $\left.v\right|_{\Gamma}$. If it is clear from the context, we will not distinguish $v$ from $\left.v\right|_{\Gamma}$. The meaning of $\left.v\right|_{\Gamma_{D}}$ is similar.

In general, for a Hilbert space $X$, the adjoint space is denoted by $X^{*}$, and $X^{N}$ denotes the set of vector $v=\left(v_{1}, \ldots, v_{N}\right), v_{j} \in X$. For vector functions, we use same symbol to indicate their inner product and norm; $(\cdot, \cdot)_{X}=(\cdot, \cdot)_{X^{N}}$ and $\|\cdot\|_{X}=\|\cdot\|_{X^{N}}$.

We use closed subspaces of $H^{1}(\Omega)^{N}$ :

$$
\begin{aligned}
& K= \begin{cases}H^{1}(\Omega)^{N} & \text { if } \Gamma_{D}=\emptyset, \\
\left\{v \in H^{1}(\Omega)^{N}|v|_{\Gamma_{D}}=0\right\} & \text { if } \Gamma_{D} \neq \emptyset ;\end{cases} \\
& K_{\tau}=\left\{v \in K\left|v_{\tau}\right|_{\Gamma}=0\right\} ; \quad \hat{K}_{\tau}=\left\{v \in K_{\tau} \mid \operatorname{div} v=0 \text { in } \Omega\right\} ; \\
& K_{n}=\left\{v \in K\left|v_{n}\right|_{\Gamma}=0\right\} ; \quad \hat{K}_{n}=\left\{v \in K_{n} \mid \operatorname{div} v=0 \text { in } \Omega\right\} .
\end{aligned}
$$

If $\Gamma_{D} \neq \emptyset,\|\cdot\|_{1}$ is equivalent to Dirichlet's norm $\|\nabla \cdot\|$ in $K$ by Poincaré's inequality. We shall not emphasise this in what follows.

Let $\psi$ be a proper $(\psi \not \equiv \infty)$ lower semi-continuous convex function defined on $\mathbb{R}^{m}, m=1$ or $N$. Then, for any $z \in \mathbb{R}^{m}, \partial \psi(z)$ denotes the set

$$
\partial \psi(z)=\left\{h \in \mathbb{R}^{m} \mid \psi(t)-\psi(z) \geq h \cdot(t-z) \forall t \in \mathbb{R}^{m}\right\}
$$

which is called the subdifferential of $\psi$ at $z$. It is easy to see that the right-hand side of (1.5) coincides with $\partial \psi(z)$, when $\psi(z)=|z|$ for $z \in \mathbb{R}^{m}$.

The symbol $C$ denotes various generic positive constant depending on $\Omega$. When we need to specify the dependence of other parameters $q_{1}, \ldots, q_{M}$, which may not be numbers, we shall write as $C=C\left(q_{1}, \ldots, q_{M}\right)$.

(B) Bilinear forms. We introduce a bilinear form on $H^{1}(\Omega)^{N} \times H^{1}(\Omega)^{N}$ defined as

$$
a_{\lambda}(v, w)=\lambda \int_{\Omega} v \cdot w d x+\frac{1}{2} \int_{\Omega} e_{i j}(v) e_{i j}(w) d x \quad\left(v, w \in H^{1}(\Omega)^{N}\right)
$$

for $\lambda \geq 0$. Here and hereafter the summation convection is employed. We put

$$
a(v, w)=a_{0}(v, w) .
$$

Clearly $a_{\lambda}$ is continuous on $H^{1}(\Omega)^{N} \times H^{1}(\Omega)^{N}$ :

$$
\left|a_{\lambda}(v, w)\right| \leq C(\lambda)\|v\|_{1}\|w\|_{1} \quad\left(\forall v, w \in H^{1}(\Omega)^{N}\right) .
$$


If $\lambda>0, a_{\lambda}$ is coercive on $H^{1}(\Omega)^{N} \times H^{1}(\Omega)^{N}$, that is,

$$
a_{\lambda}(v, v) \geq C(\lambda)\|v\|_{1}^{2} \quad\left(\forall v \in H^{1}(\Omega)^{N}\right) .
$$

In fact, (2.3) is a consequence of Korn's inequality (for example [8], [30])

$$
\int_{\Omega} e_{i j}(v) e_{i j}(v) d x+\int_{\Omega}|v|^{2} d x \geq C\|v\|_{1}^{2} \quad\left(\forall v \in H^{1}(\Omega)^{N}\right) .
$$

In the case of $\lambda=0$, we assume $\Gamma_{D} \neq \emptyset$. Then, it holds that

$$
\int_{\Omega} e_{i j}(v) e_{i j}(v) d x \geq C\|v\|_{1}^{2} \quad(\forall v \in K) .
$$

(See, for example, Theorem 6.3-4 of Ciarlet [30]. Although the proof only in the case of $N=3$ is explicitly stated there, it is valid in the case of $N=2$, too.) This, together with Poincaré's inequality, implies

$$
a(v, v) \geq C\|v\|_{1}^{2} \quad(\forall v \in K) .
$$

We shall also use a continuous bilinear form on $H^{1}(\Omega)^{N} \times L^{2}(\Omega)$

$$
b(v, \chi)=-\int_{\Omega} \chi \operatorname{div} v d x \quad\left(v \in H^{1}(\Omega)^{N}, \chi \in L^{2}(\Omega)\right) .
$$

(C) Green's formula. If a smooth vector field $u$ and a smooth scalar field $p$ solve (1.1), then by integration by parts,

$$
a_{\lambda}(u, \varphi)+b(\varphi, p)=\int_{\partial \Omega} \sigma \cdot \varphi d s+(f, \varphi) \quad\left(\forall \varphi \in H^{1}(\Omega)^{N}\right) .
$$

In particular,

$$
\begin{array}{ll}
a_{\lambda}(u, \varphi)+b(\varphi, p)=\int_{\Gamma} \sigma_{n} \varphi_{n} d s+(f, \varphi) & \left(\forall \varphi \in K_{\tau}\right), \\
a_{\lambda}(u, \varphi)+b(\varphi, p)=\int_{\Gamma} \sigma_{\tau} \cdot \varphi_{\tau} d s+(f, \varphi) & \left(\forall \varphi \in K_{n}\right) .
\end{array}
$$

Variational inequalities (Pr. WLF) and (Pr. WSF) which will appeared in the subsequent sections are based on these identities and the definition of subdifferentials.

(D) Lemmas. With the bilinear form $b$, we associate the bounded linear operator $B_{\tau}: L^{2}(\Omega) \rightarrow K_{\tau}$ defined as

$$
\left(B_{\tau} \chi, v\right)_{1}=b(v, \chi) \quad\left(\forall \chi \in L^{2}(\Omega), \forall v \in K_{\tau}\right) .
$$

Clearly we have $\left\|B_{\tau} \chi\right\|_{1} \leq\|\chi\|$ for all $\chi \in L^{2}(\Omega)$. 
Lemma 2.1. The range $R\left(B_{\tau}\right)$ of $B_{\tau}$ is a closed set in $K_{\tau}$. Moreover we have the orthogonal decomposition $K_{\tau}=R\left(B_{\tau}\right) \bigoplus \hat{K}_{\tau}$.

The proof of this lemma depends on

Lemma 2.2. Any $\chi \in L^{2}(\Omega)$ admits $w \in K_{\tau}$ satisfying $\operatorname{div} w=\chi$ in $\Omega$ and $\|w\|_{1} \leq C\|\chi\|$.

Proof. We only state the case of $\Gamma_{D} \neq \emptyset$. Firstly we assume that $\bar{\Gamma} \cap \overline{\Gamma_{D}}=$ $\emptyset$. Let $B$ an open ball which includes $\bar{\Omega}$ and take $v \in H^{2}(\Omega)$ satisfying

$$
\Delta v=\left\{\left.\begin{array}{ll}
\chi & \text { in } \Omega \\
0 & \text { in } B \backslash \Omega,
\end{array} \quad v\right|_{\partial B}=0, \quad\|v\|_{2, B} \leq C\|\chi\| .\right.
$$

Put

$$
b=\left\{\begin{array}{ll}
\left.(\nabla v)\right|_{\Gamma}+c n & \text { on } \Gamma \\
\left.(\nabla v)\right|_{\Gamma_{D}} & \text { on } \Gamma_{D}
\end{array} \quad \text { with } \quad c=-\frac{1}{|\Gamma|} \int_{\partial \Omega} \frac{\partial v}{\partial n} d s\right.
$$

where $|\Gamma|$ denotes the Lebesgue measure of $\Gamma$. Then $b \in H^{1 / 2}(\partial \Omega)^{N}$ and it satisfies

$$
\int_{\partial \Omega} b_{n} d s=0 \quad \text { and } \quad\|b\|_{1 / 2, \partial \Omega} \leq C\|\nabla v\|_{1, B} \leq C\|v\|_{2, B} \leq C\|\chi\| .
$$

Hence, as is well-known (for example Lemma I-3.2 of [17]), there is a $\psi \in$ $H^{1}(\Omega)^{N}$ such that $\operatorname{div} \psi=0$ in $\Omega,\left.\psi\right|_{\partial \Omega}=b$ and $\|\psi\|_{1} \leq C\|b\|_{1 / 2, \partial \Omega}$. We set $w=\nabla v-\psi$, which is the desired function. In fact, $\operatorname{div} w=0,\left.w\right|_{\Gamma_{D}}=0$, $\left.w_{\tau}\right|_{\Gamma}=c n-n c=0$, and $\|w\|_{1} \leq\|\nabla v\|_{1}+\|\psi\|_{1} \leq C\|v\|_{2, B} \leq C\|\chi\|$.

Next we consider the case of $\bar{\Gamma} \cap \overline{\Gamma_{D}} \neq \emptyset$. Fix $\tilde{\varphi} \in C^{\infty}(\bar{\Omega})$ satisfying $\left.\tilde{\varphi}\right|_{\Gamma_{D}}=0$ and $\left.\tilde{\varphi}\right|_{\Gamma}>0$. Then put $\varphi=\left.\tilde{\varphi}\right|_{\partial \Omega}$. In this case, we define

$$
b=\left\{\begin{array}{ll}
\left.(\nabla v)\right|_{\Gamma}+c n \varphi & \text { on } \Gamma \\
\left.(\nabla v)\right|_{\Gamma_{D}} & \text { on } \Gamma_{D}
\end{array} \quad \text { with } \quad c=-\left(\int_{\Gamma} \varphi d s\right)^{-1} \int_{\partial \Omega} \frac{\partial v}{\partial n} d s .\right.
$$

The rest is the same as before.

Proof of Lemma 2.1. Let $\chi \in L^{2}(\Omega)$. By Lemma 2.2, we can take $w \in K_{\tau}$ such that $\operatorname{div} w=q$ and $\|w\|_{1} \leq C\|\chi\|$. Substituting $v=w$ into (2.10), we obtain

$$
\|\chi\|^{2} \leq\left\|B_{\tau} \chi\right\| \cdot\|w\| \leq C\left\|B_{\tau} \chi\right\| \cdot\|\chi\|
$$


Hence it follows from the closed range theorem that $R\left(B_{\tau}\right)$ is a closed set. To prove the second assertion, it suffices to see $R\left(B_{\tau}\right)^{\perp}=\hat{K}_{\tau}$, where $R\left(B_{\tau}\right)^{\perp}$ stands for the orthogonal complement of $R\left(B_{\tau}\right)$ in $K_{\tau}$. Let $\varphi \in \hat{K}_{\tau}$. Then $\left(B_{\tau} \chi, \varphi\right)_{1}=(\chi, \operatorname{div} \varphi)=0$ for all $\chi \in L^{2}(\Omega)$ so that $\varphi \in R\left(B_{\tau}\right)^{\perp}$. Conversely, let $\varphi \in R\left(B_{\tau}\right)^{\perp}$. We have $0=\left(B_{\tau} \chi, \varphi\right)=(\chi \text {, div } \varphi)_{1}$ for all $\chi \in L^{2}(\Omega)$. Taking $\chi=\operatorname{div} \varphi$, we obtain $\operatorname{div} \varphi=0$ a.e. in $\Omega$.

Remark. Lemma 2.2 guarantees the inf-sup condition

$$
\exists \beta>0: \quad \inf _{\chi \in L^{2}(\Omega)} \sup _{v \in K_{\tau}} \frac{b(v, \chi)}{\|v\|_{1}\|\chi\|} \geq \beta,
$$

which will be used in $\S 6$. In fact, for $\chi \in X$, we take $w \in K_{\tau}$ such as described in Lemma 2.2. Then

$$
\sup _{v \in K_{\tau}} \frac{b(v, \chi)}{\|v\|_{1}} \geq \frac{b(w, \chi)}{\|w\|_{1}} \geq \frac{\|\chi\|^{2}}{\|w\|_{1}} \geq \frac{1}{C}\|\chi\| .
$$

Next, let $B_{n}: L_{0}^{2}(\Omega) \rightarrow K_{n}$ be the bounded linear operator associated with $b$ defined by

$$
\left(B_{n} \chi, v\right)_{1}=b(v, \chi) \quad\left(\forall \chi \in L_{0}^{2}(\Omega), \forall v \in K_{n}\right) .
$$

Then we have

Lemma 2.3. $R\left(B_{n}\right)$ is closed, and $K_{n}=R\left(B_{n}\right) \bigoplus \hat{K}_{n}$.

The proof, which we omit, depends on the following well-known result; For example, Corollary I-2.4 of [17].

Lemma 2.4. Any $\chi \in L_{0}^{2}(\Omega)$ admits $w \in H_{0}^{1}(\Omega)^{N}$ satisfying $\operatorname{div} w=\chi$ in $\Omega$ and $\|w\|_{1} \leq C\|\chi\|$.

\section{$\S 3 . \quad$ Leak Problem of Friction Type. Variational Inequality}

We introduce

$$
j(\eta)=\int_{\Gamma} g|\eta| d s \quad\left(\eta \in H^{1 / 2}(\Gamma)\right),
$$

which we call a friction functional. Then, as a weak form of (Pr. LF), we consider the following variational inequality: 
(Pr. WLF). Find $u \in K_{\tau}$ and $p \in L^{2}(\Omega)$ satisfying

$$
\begin{aligned}
& a_{\lambda}(u, v-u)+b(v-u, p) \\
& +j\left(v_{n}\right)-j\left(u_{n}\right) \geq(f, v-u) \quad\left(\forall v \in K_{\tau}\right), \\
& b(u, \chi)=0 \\
& \left(\forall \chi \in L^{2}(\Omega)\right) \text {. }
\end{aligned}
$$

Fujita [9] proved the following

Proposition 3.1. Let $\lambda=0$ and $\bar{\Gamma} \cap \bar{\Gamma}_{D}=\emptyset$. Suppose that $\Gamma_{D} \neq \emptyset$,

$$
f \in L^{2}(\Omega)^{N}, \quad g \in L^{2}(\Gamma)
$$

and $g>0$ a.e.in $\Gamma$. Then there is a solution $\{u, p\}$ of (Pr. WLF). The velocity $u$ is unique, but the uniqueness of $p$ depends on cases.

Remark. We can extend Proposition 3.1 to the case where $\lambda \geq 0$ and $\bar{\Gamma} \cap \bar{\Gamma}_{D} \neq \emptyset$ in the almost same way as in [9]. However, in the argument of [9], the positively of $g$ is essential to treat (Pr. WLF).

A solution $\{u, p\}$ of (Pr. WLF) is actually a strong solution of (Pr. LF) under some reasonable assumptions. In order to prove it, we approximate a solution $\{u, p\}$ of the inequality (3.2) by solutions $\left\{u_{\varepsilon}, p_{\varepsilon}\right\}$ of equations which are obtained by replacing $j$ by a regularized functional $j_{\varepsilon}$. Then the regularity of $\left\{u_{\varepsilon}, p_{\varepsilon}\right\}$ is studied.

Let $\varepsilon>0$. We define a regularization of $j$ as

$$
j_{\varepsilon}(\eta)=\int_{\Gamma} g \rho_{\varepsilon}(\eta) d s \quad\left(\eta \in H^{1 / 2}(\Gamma)\right),
$$

where $\rho_{\varepsilon}$ denotes the Yosida regularization of $\rho(z)=|z|(z \in \mathbb{R})$;

$$
\rho_{\varepsilon}(z)=\left(\frac{1-(1+\varepsilon \rho)^{-1}}{\varepsilon}\right)(z)= \begin{cases}z-\varepsilon / 2 & (z>\varepsilon) \\ z^{2} /(2 \varepsilon) & (-\varepsilon \leq z \leq \varepsilon) \\ -z+\varepsilon / 2 & (z<-\varepsilon) .\end{cases}
$$

Actually, $j_{\varepsilon}$ is an approximation of $j$ in the sense that

$$
\left|j_{\varepsilon}(\eta)-j(\eta)\right| \leq \frac{\varepsilon}{2}\|g\|_{L^{1}(\Gamma)} \quad\left(\eta \in H^{1 / 2}(\Gamma)\right) .
$$

Moreover, $j_{\varepsilon}$ is Gâteaux differentiable in $H^{1 / 2}(\Gamma)$, namely

$$
\lim _{h \rightarrow 0} \frac{1}{h}\left[j_{\varepsilon}(\eta+h \xi)-j_{\varepsilon}(\eta)\right]=\int_{\Gamma} g \alpha_{\varepsilon}(\eta) \xi d s \quad\left(\eta, \xi \in H^{1 / 2}(\Gamma)\right),
$$


where

$$
\alpha_{\varepsilon}(z)=\partial \rho_{\varepsilon}(z)= \begin{cases}1 & (z>\varepsilon) \\ z / \varepsilon & (-\varepsilon \leq z \leq \varepsilon) \\ -1 & (z<-\varepsilon) .\end{cases}
$$

Remark. Since $\rho$ is proper, convex and lower semi-continuous, $\alpha \equiv \partial \rho$ is a maximal monotone graph on $\mathbb{R}$. The function $\alpha_{\varepsilon}$ is nothing but the Yosida regularization of $\alpha ; \alpha_{\varepsilon}=\left(1-(1+\varepsilon \alpha)^{-1}\right) / \varepsilon$.

We state a regularized problem to (Pr. WLF):

$\left(\operatorname{Pr} . \mathrm{WLF}_{\varepsilon}\right)$. Find $u_{\varepsilon} \in K_{\tau}$ and $p_{\varepsilon} \in L^{2}(\Omega)$ satisfying

$$
\begin{array}{ll}
a_{\lambda}\left(u_{\varepsilon}, v-u_{\varepsilon}\right)+b\left(v-u_{\varepsilon}, p_{\varepsilon}\right) & \\
\quad+j_{\varepsilon}\left(v_{n}\right)-j_{\varepsilon}\left(u_{\varepsilon, n}\right) \geq\left(f, v-u_{\varepsilon}\right) & \left(\forall v \in K_{\tau}\right), \\
b\left(u_{\varepsilon}, \chi\right)=0 \quad & \left(\forall \chi \in L^{2}(\Omega)\right) .
\end{array}
$$

Lemma 3.1. Let $\lambda \geq 0$ and $\varepsilon>0$. Suppose that $\Gamma_{D} \neq \emptyset$ if $\lambda=0$. Assume that (3.4) and $g \geq 0$ a.e.in $\Gamma$. Then $\left(\operatorname{Pr} . \mathrm{WLF}_{\varepsilon}\right)$ admits a unique solution $\left\{u_{\varepsilon}, p_{\varepsilon}\right\} \in K_{\tau} \times L^{2}(\Omega)$ satisfying

$$
\left\|u_{\varepsilon}\right\|_{1}+\left\|p_{\varepsilon}\right\| \leq C(\lambda)\left(\|f\|+\|g\|_{\Gamma}\right) .
$$

The proof is divided into the following two lemmas.

Lemma 3.2. Under the same assumption of Lemma 3.1, there is a unique solution $\left\{u_{\varepsilon}, p_{\varepsilon}\right\} \in K_{\tau} \times L^{2}(\Omega)$ of

$$
\begin{array}{ll}
a_{\lambda}\left(u_{\varepsilon}, \varphi\right)+b\left(\varphi, p_{\varepsilon}\right)+\int_{\Gamma} g \alpha_{\varepsilon}\left(u_{\varepsilon, n}\right) \varphi_{n} d s=(f, \varphi) & \left(\forall \varphi \in K_{\tau}\right), \\
b\left(u_{\varepsilon}, \chi\right)=0 & \left(\forall \chi \in L^{2}(\Omega)\right),
\end{array}
$$

and $\left\{u_{\varepsilon}, p_{\varepsilon}\right\}$ satisfies $(3.9)$.

Lemma 3.3. Suppose that the same assumption of Lemma 3.1 holds. Then $\left\{u_{\varepsilon}, p_{\varepsilon}\right\} \in K_{\tau} \times L^{2}(\Omega)$ is a solution of $\left(\operatorname{Pr} . \mathrm{WLF}_{\varepsilon}\right)$ if and only if $\left\{u_{\varepsilon}, p_{\varepsilon}\right\}$ is a one of (3.10) and (3.11).

Proof of Lemma 3.2. We shall drop the subscript $\varepsilon$ for the sake of simplicity and put $V=\hat{K}_{\tau}$. We start with the minimization problem:

$$
\text { Find } u \in V: \quad J(u) \leq J(v) \quad(\forall v \in V),
$$


where

$$
J(v)=\frac{1}{2} a_{\lambda}(v, v)-(f, v)+j\left(v_{n}\right)
$$

Since $j$ is a proper, convex, and lower semi-continuous functional, from standard theory of convex analysis (for example, Lemma I-4.1 of [14]), (3.12) admits a unique solution $u$ which is characterized by

$$
a_{\lambda}(u, v-u)+j\left(v_{n}\right)-j\left(u_{n}\right) \geq(f, v-u), \quad(\forall v \in V) .
$$

Substituting into (3.13) $v=u \pm t \psi$ with arbitrary $\psi \in V, t>0$ and letting $t \downarrow 0$, we obtain by (3.6)

$$
a_{\lambda}(u, \psi)+\int_{\Gamma} g \alpha\left(u_{n}\right) \psi_{n} d s=(f, \psi), \quad(\forall \psi \in V) .
$$

In order to prove the existence of $p$ associated with $u$, we introduce a linear functional on $K_{\tau}$ by setting

$$
F(\varphi)=a_{\lambda}(u, \varphi)+\int_{\Gamma} g \alpha\left(u_{n}\right) \varphi_{n} d s-(f, \varphi), \quad\left(\varphi \in K_{\tau}\right)
$$

It is bounded. In particular,

$$
|F(\varphi)| \leq C(\lambda)\left(\|u\|_{1}+\|g\|_{0, \Gamma}+\|f\|\right)\|\varphi\|_{1}, \quad\left(\forall \varphi \in K_{\tau}\right)
$$

because of $\left|\alpha\left(u_{n}\right)\right| \leq 1$.

Let $B_{\tau}$ be the bounded linear operator associated with $b$ defined as (2.10). We apply Riesz's representation theorem to $F$ on a closed subspace $R\left(B_{\tau}\right)$ of $K_{\tau}$. Thus we have $w \in R\left(B_{\tau}\right)$ such that $F(v)=(w, v)_{1}$ for any $v \in R\left(B_{\tau}\right)$. Furthermore, by the definition of $B_{\tau}$, there is a unique $p \in L^{2}(\Omega)$ satisfying $F(v)=(p, \operatorname{div} v)_{1}$ for any $v \in R\left(B_{\tau}\right)$.

Now let $\varphi \in K_{\tau}$ and, according to Lemma 2.1, we decompose $\varphi$ as $\varphi=$ $v+\psi$, where $v \in R\left(B_{\tau}\right)$ and $\psi \in V$. Then, by virtue of (3.14),

$$
F(\varphi)=F(v)+F(\psi)=F(v)=(p, \operatorname{div} v)=(p, \operatorname{div} \varphi),
$$

which means (3.10).

Next we derive (3.9). Substituting $\phi=u$ into (3.10), we get

$$
C(\lambda)\|u\|_{1}^{2}-C\|g\|_{0, \Gamma}\|u\|_{1} \leq C\|f\| \cdot\|u\|_{1} .
$$


Hence we have $\|u\|_{1} \leq C(\lambda)\left(\|g\|_{0, \Gamma}+\|f\|\right)$. We again take $w \in K_{\tau}$ such that $\operatorname{div} w=p$ and $\|w\|_{1} \leq C\|p\|$. Then by choosing $\varphi=w$ in (3.10), we obtain

$$
\begin{aligned}
\|p\|^{2} & \leq a_{\lambda}(u, w)+\int_{\Gamma} g \alpha\left(u_{n}\right) w_{n}-(f, w) \\
& \leq C(\lambda)\left(\|u\|_{1}\|w\|_{1}+\|g\|_{0, \Gamma}\|w\|_{1}+\|f\| \cdot\|w\|_{1}\right) \\
& \leq C(\lambda)\left(\|u\|_{1}+\|g\|_{0, \Gamma}+\|f\|\right)\|p\| .
\end{aligned}
$$

This completes the proof of Lemma 3.2.

Proof of Lemma 3.3. We have by (3.6) and the convexity of $j_{\varepsilon}$

$$
\int_{\Gamma} g \alpha_{\varepsilon}\left(v_{n}\right)\left(\varphi_{n}-v_{n}\right) d s \leq j_{\varepsilon}\left(\varphi_{n}\right)-j_{\varepsilon}\left(v_{n}\right) \quad\left(\forall v, \varphi \in H^{1}(\Omega)^{N}\right) .
$$

From this, we see that a solution $\left\{u_{\varepsilon}, p_{\varepsilon}\right\}$ of (3.10) with (3.11) also solves (Pr. $\mathrm{WLF}_{\varepsilon}$ ). To check the converse assertion, in (3.7), we take $v=u_{\varepsilon} \pm t \psi$ with $\psi \in K_{\tau}, t>0$. Then let $t \downarrow 0$ to obtain the result.

\section{§4. Leak Problem of Friction Type. Regularity}

In this section, we firstly study the regularity of a solution to $\left(\operatorname{Pr} . \mathrm{WLF}_{\varepsilon}\right)$ and then give the proof of Theorem 1.1.

Lemma 4.1. Let $\lambda \geq 0$ and suppose (1.6), (1.7), (1.9), and (1.10). Let $\varepsilon>0$ and let $\left\{u_{\varepsilon}, p_{\varepsilon}\right\} \in K_{\tau} \times L^{2}(\Omega)$ solve

$$
\begin{array}{ll}
a_{\lambda}\left(u_{\varepsilon}, \varphi\right)+b\left(\varphi, p_{\varepsilon}\right)+\int_{\Gamma} g \alpha_{\varepsilon}\left(u_{\varepsilon, n}\right) \varphi_{n} d s=(f, \varphi) & \left(\forall \varphi \in K_{\tau}\right), \\
b\left(u_{\varepsilon}, \chi\right)=0 & \left(\forall \chi \in L^{2}(\Omega)\right) .
\end{array}
$$

Then $u_{\varepsilon} \in H^{2}(\Omega)^{N}, p_{\varepsilon} \in H^{1}(\Omega)$ and they satisfy

$$
\begin{array}{ll}
\lambda u_{\varepsilon}-\Delta u_{\varepsilon}+\nabla p_{\varepsilon}=f, \quad \operatorname{div} u_{\varepsilon}=0 & \text { in } \Omega, \\
u_{\varepsilon}=0, & \text { on } \Gamma_{D}, \\
u_{\varepsilon, \tau}=0, \quad-\sigma_{n}\left(u_{\varepsilon}, p_{\varepsilon}\right)=g \alpha_{\varepsilon}\left(u_{\varepsilon, n}\right) & \text { on } \Gamma .
\end{array}
$$

Moreover there is a constant $C>0$ independent of $\varepsilon$ and $\lambda$ such that

$$
\left\|u_{\varepsilon}\right\|_{2}+\left\|p_{\varepsilon}\right\|_{1} \leq C\left(\|f\|+\|g\|_{1 / 2, \Gamma}+\left\|u_{\varepsilon}\right\|_{1}+\left\|p_{\varepsilon}\right\|\right) .
$$


Remark. Inequality (4.6), together with (3.9), implies

$$
\left\|u_{\varepsilon}\right\|_{2}+\left\|p_{\varepsilon}\right\|_{1} \leq C(\lambda)\left(\|f\|+\|g\|_{1 / 2, \Gamma}\right) .
$$

That is, $\left\|u_{\varepsilon}\right\|_{2}$ and $\left\|p_{\varepsilon}\right\|_{1}$ are bounded sequences as $\varepsilon \downarrow 0$.

Proof of Lemma 4.1. The regularity in interior and near $\Gamma_{D}$ is well-known; for example [7] and [22]. Specifically, let $k \geq 0$ and $\omega \subset \Omega$ be an open set subject to

(I) $\operatorname{dist}(\bar{\omega}, \partial \Omega) \geq \delta>0(\delta$ : constant $)$;

or (II) $\omega \cap \Gamma_{D} \neq \emptyset, \omega \cap \Gamma=\emptyset, \Gamma_{D}$ is of class $C^{k+2}$

then we have

$$
u \in H^{k+2}(\bar{\omega})^{N}, p \in H^{k+1}(\bar{\omega}), \quad\|u\|_{k+2, \bar{\omega}}+\|p\|_{k+1, \bar{\omega}} \leq C(\lambda, \bar{\omega})\|f\|_{k, \bar{\omega}}
$$

if $f \in H^{k}(\Omega)^{N}$. Therefore it suffices to study the regularity near $\Gamma$. Let $x_{0} \in \Gamma$ and $U_{0} \subset \mathbb{R}^{N}$ be a neighbourhood of $x_{0}$. Taking a cut-off function $\theta \in C^{\infty}\left(\mathbb{R}^{N}\right)$ subject to

$$
0 \leq \theta \leq 1, \quad \operatorname{supp} \theta \subset U_{0}, \quad \operatorname{supp} \theta \cap\left(\mathbb{R}^{N} \backslash \bar{\Omega}\right) \neq \emptyset .
$$

Substituting $\theta^{2} \varphi$ and $\theta^{2} \chi$, where $\varphi \in K_{\tau}$ and $\chi \in L^{2}(\Omega)$, into (4.1) and (4.2) as test functions, we obtain

$$
\begin{array}{lll}
\text { (4.9) } a_{\lambda}\left(\theta^{2} u, \varphi\right)+b\left(\varphi, \theta^{2} p\right)+\int_{\Gamma}\left(\theta^{2} g\right) \alpha\left(u_{n}\right) \varphi_{n} d s & \\
=\left(\theta^{2} f, \varphi\right)+F^{*}(\varphi), & \left(\forall \varphi \in K_{\tau}\right), \\
\text { (4.10) } b\left(\theta^{2} u, \chi\right)=G^{*}(\chi), & \left(\forall \chi \in L^{2}(\Omega)\right),
\end{array}
$$

where

$$
\begin{aligned}
F^{*}(\varphi) & =\frac{1}{2} \int_{\Omega}\left[\left(u_{i} \frac{\partial \theta^{2}}{\partial x_{j}}+\frac{\partial \theta^{2}}{\partial x_{i}} u_{j}\right) e_{i j}(\varphi)\right. \\
& \left.-e_{i j}(u)\left(\varphi_{i} \frac{\partial \theta^{2}}{\partial x_{j}}+\frac{\partial \theta^{2}}{\partial x_{i}} \varphi_{j}\right)\right] d x+\int_{\Omega} p \varphi_{i} \frac{\partial \theta^{2}}{\partial x_{i}} d x \\
G^{*}(\chi) & =-\int_{\Omega} \chi u_{i} \frac{\partial \theta^{2}}{\partial x_{i}} d x
\end{aligned}
$$

Here and hereafter, we drop $\varepsilon$ for simplicity. We take $R>0, U \subset U_{0}$ and a one-to-one mapping $\Phi=\left(\Phi_{1}, \ldots, \Phi_{N}\right)$ from $U$ onto $\tilde{U} \subset \mathbb{R}_{y}^{N}$ enjoying the following properties (see, for example, §I-2 of Wolka [29]): 
1. $\Phi$ is a $C^{3}$-diffeomorphism;

2. $\Phi\left(x_{0}\right)=0$;

3. $\Phi(U \cap \Omega)=Q_{R} \equiv\left\{y=\left(y^{\prime}, y_{N}\right) \in \mathbb{R}^{N-1} \times \mathbb{R}|| y^{\prime} \mid<R, 0<y_{N}<R\right\}$;

4. $\Phi(U \cap \Gamma)=S_{R} \equiv\left\{y=\left(y^{\prime}, y_{N}\right) \in \mathbb{R}^{N-1} \times \mathbb{R}|| y^{\prime} \mid<R, y_{N}=0\right\}$;

5. $\frac{\partial \Phi_{N}}{\partial x_{j}}=\frac{\partial \Phi_{j}}{\partial x_{N}}=0$ and $\frac{\partial \Phi_{N}}{\partial x_{N}}=-1$ on $U \cap \Gamma(j=1, \ldots, N-1)$;

6. $\Phi: n(x) \mapsto \tilde{n}(y) \equiv(0, \ldots, 0,-1)$ for $x \in U \cap \Gamma$.

We introduce

$$
\begin{aligned}
& K\left(Q_{R}\right)=\left\{\varphi \in H^{1}\left(Q_{R}\right)^{N} \mid \varphi(y)=0 \text { for }|y|=R, y_{N}=R\right\} \\
& K_{\tau}\left(Q_{R}\right)=\left\{\varphi \in K\left(Q_{R}\right) \mid \varphi_{j}=0 \text { on } S_{R}(j=1, \ldots, N-1)\right\}
\end{aligned}
$$

and set $y=\Phi(x)=\left(\Phi_{1}(x), \ldots, \Phi_{N}(x)\right)$,

$$
\tilde{u}(y)=\left(\theta^{2} u\right)(x), \quad \tilde{p}(y)=\left(\theta^{2} p\right)(x)
$$

and

$$
\tilde{f}(y)=\left(\theta^{2} f\right)(x), \quad \tilde{g}(y)=\left(\theta^{2} g\right)(x) .
$$

It should be kept in mind that for any $\tilde{\varphi} \in K_{\tau}\left(Q_{R}\right)$ the function on $\Omega$ defined as

$$
\varphi(x)= \begin{cases}\tilde{\varphi}(y) & \text { for } y \in Q_{R} \\ 0 & \text { otherwise }\end{cases}
$$

is in $K_{\tau}$. Hence we obtain by (4.9) and (4.10)

$$
\begin{array}{rlr}
\tilde{a}_{\lambda}(\tilde{u}, \tilde{\varphi})+\tilde{b}(\tilde{\varphi}, \tilde{p})- & \int_{S_{R}} \tilde{g} \alpha\left(-\tilde{u}_{N}\right) \tilde{\varphi}_{N} d y^{\prime} \\
& =\int_{Q_{R}} \tilde{f} \cdot \tilde{\varphi}|\operatorname{Jac} \Phi| d y+\tilde{F}(\tilde{\varphi}), \quad\left(\forall \tilde{\varphi} \in K_{\tau}\left(Q_{R}\right)\right), \\
\tilde{b}(\tilde{u}, \tilde{\chi})=\tilde{G}(\tilde{\chi}), & & \left(\forall \tilde{\chi} \in L^{2}\left(Q_{R}\right)\right) .
\end{array}
$$

(4.14) $\tilde{b}(\tilde{u}, \tilde{\chi})=\tilde{G}(\tilde{\chi})$, 
Here we have put:

$$
\begin{aligned}
& \tilde{a}_{\lambda}(\tilde{u}, \tilde{\varphi})=\lambda \int_{Q_{R}} \tilde{u} \cdot \tilde{\varphi} d y+\frac{1}{2} \int_{Q_{R}} \tilde{e}_{i j}(\tilde{u}) \tilde{e}_{i j}(\tilde{\varphi}) d y \\
& \tilde{e}_{i j}(\tilde{u}) \tilde{e}_{i j}(\tilde{\varphi})=\left(\frac{\partial \Phi_{k}}{\partial x_{j}} \frac{\partial \tilde{u}_{i}}{\partial y_{k}}+\frac{\partial \Phi_{k}}{\partial x_{i}} \frac{\partial \tilde{u}_{j}}{\partial y_{k}}\right)\left(\frac{\partial \Phi_{l}}{\partial x_{j}} \frac{\partial \tilde{\varphi}_{i}}{\partial y_{l}}+\frac{\partial \Phi_{l}}{\partial x_{i}} \frac{\partial \tilde{\varphi}_{j}}{\partial y_{l}}\right) ; \\
& \tilde{b}(\tilde{\varphi}, \tilde{\chi})=-\int_{Q_{R}} \tilde{\chi} \frac{\partial \Phi_{k}}{\partial x_{i}} \frac{\partial \tilde{\varphi}_{i}}{\partial y_{k}}|\mathrm{Jac} \Phi| d y ; \\
& \tilde{F}(\tilde{\varphi})=\int_{Q_{R}}\left[\sum_{i, j, k}\left(d_{i j k}^{(1)} \tilde{u}_{i} \frac{\partial \tilde{\varphi}_{i}}{\partial y_{k}}+d_{i j k}^{(2)} \frac{\partial \tilde{u}_{i}}{\partial y_{j}} \tilde{\varphi}_{k}\right)\right. \\
& \left.+\sum_{i, j} d_{i j}^{(3)} \tilde{u}_{i} \tilde{\varphi}_{j}+\sum_{i} d_{i}^{(4)} \tilde{p} \tilde{\varphi}_{i}\right]|\mathrm{Jac} \Phi| d y ; \\
& \tilde{G}(\tilde{\chi})=\int_{Q_{R}} d_{i}^{(5)} \tilde{\chi} \tilde{u}_{i}|\mathrm{Jac} \Phi| d y,
\end{aligned}
$$

where $d_{i j k}^{(1)}, d_{i j k}^{(2)}, d_{i j}^{(3)}, d_{i}^{(4)}$, and $d_{i}^{(5)}$ are $C^{\infty}\left(\overline{Q_{R}}\right)$ functions composed of $\theta$ and $\partial \theta / \partial x_{l}$.

Now take $R_{1} \in(0, R)$ and put $R_{2}=R-\varepsilon$, where $\varepsilon=\left(R-R_{1}\right) / 2$. We simply write $Q_{i}=Q_{R_{i}}, S_{i}=S_{R_{i}}(i=1,2)$, and $Q=Q_{R}, S=S_{R}$. We will not distinguish $\varphi \in K\left(Q_{i}\right)$ with its zero extension $\hat{\varphi} \in K(Q)$ ( $\hat{\varphi}=\varphi$ in $Q_{i}$, $\hat{\varphi}=0$ in $\left.Q \backslash Q_{i}\right)$ for the sake of simplicity.

By re-choosing the cut-off function $\theta$ if necessary, we may assume that

$$
\tilde{u} \in K_{\tau}\left(Q_{1}\right), \quad \tilde{g} \in H_{00}^{1 / 2}\left(S_{1}\right),
$$

where

$$
H_{00}^{1 / 2}\left(S_{1}\right)=\left\{\xi \in H^{1 / 2}(\Gamma) \mid\left(R_{1}-\left|y^{\prime}\right|\right)^{-1 / 2} \xi \in L^{2}\left(S_{1}\right)\right\}
$$

which is a Hilbert space equipped with the norm

$$
\|\xi\|_{00, S_{1}}=\|\xi\|_{H_{00}^{1 / 2}\left(S_{1}\right)}=\left[\|\xi\|_{Q_{1}}^{2}+\int_{S_{1}} \frac{\xi^{2}}{R_{1}-\left|y^{\prime}\right|} d y^{\prime}\right]^{1 / 2} .
$$

We shall review a more general definition of $H_{00}^{1 / 2}$ and its properties in Appendix. At this stage we only mention that

$$
\tilde{g} \in H_{00}^{1 / 2}(S), \quad\|\tilde{g}\|_{00, S} \leq\|g\|_{1 / 2, \Gamma} .
$$

(The definition of $H_{00}^{1 / 2}(S)$ is similar.) 
Now we recall the notation of the finite difference quotients. Let $h \in \mathbb{R}$ and $\left\{e_{i}\right\}_{i=1, \ldots, N}$ be the canonical basis of $\mathbb{R}_{y}^{N}$. Shift and forward difference operators are defined by

$$
s_{h}^{i} v(y)=v\left(y+h e_{i}\right) \quad \text { and } \quad D_{h}^{i} v(y)=\frac{s_{h}^{i} v(y)-v(y)}{|h|} .
$$

It is well-known (for example [6]) that

$$
\begin{gathered}
D_{h}^{i}(u v)=u\left(D_{h}^{i} v\right)+\left(D_{h}^{i} u\right)\left(s_{h}^{i} v\right) \quad\left(u, v \in K\left(Q_{j}\right)\right), \\
\int_{Q} u \cdot D_{-h}^{i} v d y=\int_{Q}\left(D_{h}^{i}\right) u \cdot v d y \quad\left(u, v \in K\left(Q_{j}\right)\right), \\
\left\|D_{h}^{i} u\right\|_{Q} \leq\|\nabla u\|_{Q} \quad\left(u \in K\left(Q_{j}\right)\right)
\end{gathered}
$$

for $j=1,2, i=1, \ldots, N-1$ and a suitably small $h$.

In the following, for $k \geq 0, C_{k}$ denotes a positive constant depending on $\partial^{k} \Phi / \partial x_{j}^{k}$. Let $0<h<\varepsilon$. We fix $i \in\{1,2, \ldots, N-1\}$ and write $D_{ \pm h}=D_{ \pm h}^{i}$. Then $D_{h} \tilde{u} \in K_{\tau}\left(Q_{2}\right)$. By choosing

$$
\tilde{\varphi}=v \equiv D_{-h}\left(D_{h} \tilde{u}\right) \in K_{\tau}\left(Q_{1}\right),
$$

in (4.13), we obtain

$$
\begin{aligned}
& \left\|\nabla_{y}\left(D_{h} \tilde{u}\right)\right\|_{Q}^{2} \\
& \quad \leq C_{3}\left(\|\tilde{u}\|_{1, Q}+\|\tilde{p}\|_{Q}+\|\tilde{g}\|_{00, S}+\|\tilde{f}\|_{Q}\right)\left(\|\tilde{u}\|_{1, Q}+\left\|\nabla_{y}\left(D_{h} \tilde{u}\right)\right\|_{Q}\right)
\end{aligned}
$$

by making use of

$$
\begin{gathered}
\tilde{a}_{\lambda}(\tilde{u}, v) \geq C_{1}\left\|\nabla_{y}\left(D_{h} \tilde{u}\right)\right\|_{Q}^{2}-C_{2}\|\tilde{u}\|_{1, Q}\left\|\nabla_{y}\left(D_{h} \tilde{u}\right)\right\|_{Q} \\
|\tilde{b}(v, \tilde{p})| \leq C_{3}\|\tilde{p}\|_{Q_{R}}\left(\|\tilde{u}\|_{1, Q}+\left\|\nabla_{y}\left(D_{h} \tilde{u}\right)\right\|_{Q}\right) ; \\
\int_{S} \tilde{g} \alpha\left(-\tilde{u}_{N}\right) v_{N}\left(y^{\prime}, 0\right) d y^{\prime} \leq C_{2}\|\tilde{g}\|_{00, S}\left\|\nabla_{y}\left(D_{h} \tilde{u}\right)\right\|_{Q},
\end{gathered}
$$

and

$$
\begin{aligned}
& \left|\int_{Q}(\tilde{f} \cdot v)\right| \operatorname{Jac} \Phi|d y+\tilde{F}(v)| \\
& \quad \leq C_{2}\left(\|\tilde{f}\|_{Q}+\|\tilde{u}\|_{1, Q}+\|\tilde{p}\|_{Q}\right)\left(\|\tilde{u}\|_{1, Q}+\left\|\nabla_{y}\left(D_{h} \tilde{u}\right)\right\|_{Q}\right),
\end{aligned}
$$

where $\nabla_{y}\left(D_{h} \tilde{u}\right)$ denotes the tensor product. We shall verify inequalities (4.17)(4.20) later and continue the proof of Lemma 4.1. Equality (4.16) implies

$$
\left\|\nabla_{y}\left(D_{h} \tilde{u}\right)\right\|_{Q} \leq C_{3}\left(\|\tilde{u}\|_{1, Q}+\|\tilde{p}\|_{Q}+\|\tilde{g}\|_{00, S}+\|\tilde{f}\|_{Q}\right),
$$


and then letting $h \downarrow 0$,

$$
\sum_{i=1}^{N-1} \sum_{j=1}^{N}\left\|\frac{\partial}{\partial y_{i}} \frac{\partial \tilde{u}}{\partial y_{j}}\right\|_{Q} \leq C_{3}\left(\|\tilde{u}\|_{1, Q}+\|\tilde{p}\|_{Q}+\|\tilde{g}\|_{00, S}+\|\tilde{f}\|_{Q}\right) .
$$

This and the trace theorem imply that

$$
\left\|\xi_{j}\right\|_{1 / 2, S} \leq C_{3}\left(\|\tilde{u}\|_{1, Q}+\|\tilde{p}\|_{Q}+\|\tilde{g}\|_{00, S}+\|\tilde{f}\|_{Q}\right)
$$

for $j=1, \ldots, N-1$, where $\xi_{j}$ 's are defined as $\xi_{j}=\left.\left(\partial \tilde{u}_{N} / \partial y_{j}\right)\right|_{S}$. This means in particular that all tangential derivatives of $\left.\tilde{u}_{N}\right|_{S}$ belongs to $H^{1 / 2}(S)$. Therefore, $\left.\tilde{u}_{N}\right|_{S} \in H^{3 / 2}(S)$ and

$$
\left\|\left.\tilde{u}_{N}\right|_{S}\right\|_{3 / 2, S} \leq C_{3}\left(\|\tilde{u}\|_{1, Q}+\|\tilde{p}\|_{Q}+\|\tilde{g}\|_{00, S}+\|\tilde{f}\|_{Q}\right) .
$$

Summing up the above estimates, by the argument of the partition of unity, we finally have

$$
\|\beta\|_{3 / 2, \Gamma} \leq C_{3}\left(\|u\|_{1, \Omega}+\|p\|_{\Omega}+\|g\|_{1 / 2, \Gamma}+\|f\|_{\Omega}\right),
$$

where $\beta=\left.u_{n}\right|_{\Gamma}$. Therefore, in accordance with a well-known regularity result on the Dirichlet boundary value problem for the Stokes equations by Cattabriga [7], we deduce (4.6).

To verify that a solution $\left\{u_{\varepsilon}, p_{\varepsilon}\right\} \in H^{2}(\Omega)^{N} \times H^{1}(\Omega)$ of (4.1) and (4.2) also satisfies (4.3), (4.4) and (4.5) is standard.

It remains to prove inequalities (4.17), (4.18), (4.19) and (4.20).

Proof of (4.17). We have

$$
\begin{aligned}
\tilde{a}_{\lambda}(\tilde{u}, v) & =\tilde{a}_{\lambda}\left(D_{h} \tilde{u}, D_{h} \tilde{u}\right) \\
& +\int_{Q}\left(s_{h} \tilde{e}_{i j}(\tilde{u})\right)\left[\left(D_{h} \frac{\partial \Phi_{l}}{\partial x_{i}}\right) \frac{\partial\left(D_{h} \tilde{u}_{j}\right)}{\partial y_{l}}+\left(D_{h} \frac{\partial \Phi_{l}}{\partial x_{j}}\right) \frac{\partial\left(D_{h} \tilde{u}_{i}\right)}{\partial y_{l}}\right] d y .
\end{aligned}
$$

The absolute value of the second term of the right-hand side is estimated by

$$
C_{2}\|\tilde{u}\|_{1, Q}\left\|\nabla_{y}\left(D_{h} \tilde{u}\right)\right\|_{Q} .
$$

On the other hand, we have

$$
\tilde{a}_{\lambda}\left(D_{h} \tilde{u}, D_{h} \tilde{u}\right) \geq C_{1}\left\|\nabla_{y}\left(D_{h} \tilde{u}\right)\right\|_{Q}^{2},
$$

since

$$
\tilde{a}_{\lambda}(w, w) \geq C_{1} \int_{Q_{R}}|\nabla w|^{2} d y \quad(\forall w \in K(Q))
$$


which is proved in the following way. Recall the change of variables $\Phi: x \rightarrow y$ and write

$$
\hat{w}(x)= \begin{cases}w(y) & y \in Q_{R} \\ 0 & \text { otherwise. }\end{cases}
$$

Then $\hat{w} \in K$ and $\hat{w}=0$ on $\Gamma \backslash U$. (2.5) gives us

$$
\frac{1}{2} \int_{\Omega} e_{i j}(\hat{w}) e_{i j}(\hat{w}) d x \geq C \int_{\Omega}|\nabla \hat{w}|^{2} d x .
$$

Noting that there is $0<\underline{\mu}<\bar{\mu}$ such that $\underline{\mu} \leq \operatorname{Jac} \Phi \leq \bar{\mu}$, where Jac $\Phi$ denotes the Jacobian of $\left(\partial \Phi_{i} / \partial x_{j}\right)$, we can estimate as

$$
\begin{aligned}
& \frac{1}{2} \int_{\Omega} e_{i j}(\hat{w}) e_{i j}(\hat{w}) d x \\
& \leq \frac{\bar{\mu}}{2} \int_{Q_{R}} \sum_{k, l}\left(a_{k, l}^{0} \frac{\partial w}{\partial y_{k}} \frac{\partial w}{\partial y_{l}}+\sum_{\nu, \nu^{\prime}} \frac{\partial \Phi_{l}}{\partial x_{\nu}} \frac{\partial \Phi_{k}}{\partial x_{\nu^{\prime}}} \frac{\partial w_{\nu}}{\partial y_{k}} \frac{\partial w_{\nu^{\prime}}}{\partial y_{l}}\right) d y
\end{aligned}
$$

and

$$
\int_{\Omega}|\nabla \hat{w}|^{2} d x \geq \underline{\mu} \int_{Q_{R}}|\nabla w|^{2} d y .
$$

Hence we obtain (4.21).

Proof of (4.18). Putting $\eta=|\operatorname{Jac} \Phi| \partial \Phi_{k} / \partial x_{i}$, we have

$$
\begin{aligned}
\tilde{b}(v, \tilde{p}) & =\int_{Q_{R}}\left(D_{h} \tilde{p}\right)\left(\eta \frac{\partial}{\partial y_{k}} D_{h} \tilde{u}\right) d y+\int_{Q_{R}}\left(s_{h} \tilde{p}\right)\left(D_{h} \eta\right) \frac{\partial}{\partial y_{k}} D_{h} \tilde{u} d y \\
& \equiv I_{1}+I_{2} .
\end{aligned}
$$

On the other hand, substituting $\tilde{\chi}=D_{-h}\left(D_{h} \tilde{p}\right)$ into (4.14), we obtain

$$
I_{3}+I_{1}=I_{4}
$$

where

$$
\begin{aligned}
I_{3} & =-\int_{Q_{R}} \tilde{p} D_{-h}\left[\left(D_{h} \eta\right)\left(s_{h} \frac{\partial \tilde{u}_{i}}{\partial y_{k}}\right)\right] d y, \\
I_{4} & =\int_{Q_{R}} \tilde{p} D_{-h}\left[D_{h}\left(d_{i}^{(5)} \tilde{u}_{i}|\operatorname{Jac} \Phi|\right)\right] d y .
\end{aligned}
$$

It is easy to see that

$$
\begin{gathered}
\left|I_{2}\right| \leq C_{2}\|\tilde{p}\|_{Q}\left(\|\tilde{u}\|_{1, Q}+\left\|\nabla_{y}\left(D_{h} \tilde{u}\right)\right\|_{Q}\right), \\
\left|I_{3}\right|,\left|I_{4}\right| \leq C_{3}\|\tilde{p}\|_{Q}\left(\|\tilde{u}\|_{1, Q}+\left\|\nabla_{y}\left(D_{h} \tilde{u}\right)\right\|_{Q}\right) .
\end{gathered}
$$


(4.18) then follows from (4.22), (4.23), (4.24) and (4.25).

Proof of (4.20). We have

$$
\begin{aligned}
& \int_{S} \tilde{g} \alpha\left(-\tilde{u}_{N}\right) D_{-h}\left(D_{h} \tilde{u}_{N}\right) d y^{\prime} \\
& \quad=\int_{S}\left(D_{h} \tilde{g}\right)\left[s_{h} \alpha\left(-\tilde{u}_{N}\right)\right] D_{h} \tilde{u}_{N} d y^{\prime}+\int_{S} \tilde{g}\left[D_{h} \alpha\left(-\tilde{u}_{N}\right)\right] D_{h} \tilde{u}_{N} d y^{\prime} \\
& \quad \equiv J_{1}+J_{2} .
\end{aligned}
$$

Since $\alpha$ is nondecreasing, if $\tilde{u}_{N}(y+h e) \neq \tilde{u}_{N}(y)$,

$$
\left[D_{h} \alpha\left(-\tilde{u}_{N}\right)\right] D_{h} \tilde{u}_{N}=-\frac{\alpha\left(-\tilde{u}_{N}(y+h e)\right)-\alpha\left(-\tilde{u}_{N}(y)\right)}{-\tilde{u}_{N}(y+h e)-\left(-\tilde{u}_{N}(y)\right)}\left(D_{h} \tilde{u}_{N}\right)^{2} \leq 0
$$

at $y=\left(y^{\prime}, 0\right) \in S$. Hence $J_{2} \leq 0$. In order to estimate $J_{1}$, we need

Lemma 4.2. Let $0<h<\varepsilon$ and $i=1, \ldots, N-1$. Suppose $g \in$ $H_{00}^{1 / 2}\left(S_{R_{1}}\right)$. Then

$$
\left|\int_{S_{R}}\left(D_{h}^{i} g\right) \varphi d y^{\prime}\right| \leq \int_{S_{R}}\left|\left(D_{h}^{i} g\right) \varphi\right| d y^{\prime} \leq C(R)\|g\|_{00, S_{1}}\|\nabla \varphi\|_{Q_{R}}
$$

for all $\varphi \in K\left(Q_{R_{2}}\right)$.

The proof of this lemma will be given in Appendix. In view of this, by $\left|\alpha\left(-\tilde{u}_{N}\right)\right| \leq 1$, we have

$$
J_{1} \leq C_{2}\|\tilde{g}\|_{00, S_{R}}\left\|\nabla_{y} D_{h} \tilde{u}\right\|_{Q},
$$

therefore (4.20) is proved.

Proof of (4.19). It is not new; For example, see [15] or [2].

This completes the proof of Lemma 4.1.

Now we can state

Proof of Theorem 1.1. Let $\varepsilon>0$, and let $\left\{u_{\varepsilon}, p_{\varepsilon}\right\} \in K_{\tau} \times L^{2}(\Omega)$ be a unique solution of $\left(\operatorname{Pr} . \mathrm{WLF}_{\varepsilon}\right)$. By (4.7), sequences $\left\|u_{\varepsilon}\right\|_{2}$ and $\left\|p_{\varepsilon}\right\|_{1}$ are bounded as $\varepsilon \downarrow 0$. Hence, they admit a sequence $\varepsilon_{j}\left(0<\varepsilon_{j} \downarrow 0\right.$ as $\left.j \uparrow \infty\right), u \in H^{2}(\Omega)^{N} \cap K_{\tau}$, and $p \in H^{1}(\Omega)$ such that

$$
\begin{array}{ll}
u_{\varepsilon_{j}} \rightarrow u & \text { weakly in } H^{2}(\Omega)^{N}, \\
p_{\varepsilon_{j}} \rightarrow p & \text { weakly in } H^{1}(\Omega) .
\end{array}
$$


Moreover, from the trace theorem,

$$
\begin{array}{ll}
\left.\left.u_{\varepsilon_{j}}\right|_{\Gamma_{D}} \rightarrow u\right|_{\Gamma_{D}} & \text { weakly in } H^{3 / 2}\left(\Gamma_{D}\right)^{N}, \\
\left.\left.u_{\varepsilon_{j}, n}\right|_{\Gamma} \rightarrow u_{n}\right|_{\Gamma} & \text { weakly in } H^{3 / 2}(\Gamma), \\
\left.\left.\sigma_{n}\left(u_{\varepsilon_{j}}, p_{\varepsilon_{j}}\right)\right|_{\Gamma} \rightarrow \sigma_{n}(u, p)\right|_{\Gamma} & \text { weakly in } H^{1 / 2}(\Gamma) .
\end{array}
$$

By virtue of (4.3) and (4.5), $\{u, p\}$ satisfies (1.1) and (1.2). Similarly we have $\left.u_{\tau}\right|_{\Gamma}=0$. In order to see that $\{u, p\}$ satisfies the nonlinear boundary condition, we recall $\alpha_{\varepsilon}(z) \in \alpha\left((1+\varepsilon \alpha)^{-1} z\right)$ for all $z \in \mathbb{R}$, where $\alpha=\partial|\cdot|$. (This is a property of the Yosida regularization.) Hence, by the maximality of $\alpha,(4.28)$ and (4.29), we obtain

$$
-\sigma_{n}(u, p) \in g \alpha\left(u_{n}\right)
$$

On the other hand, (4.6), together with (3.9), leads to

$$
\|u\|_{2}+\|p\|_{1} \leq C\left(\|f\|+\|g\|_{1 / 2, \Gamma}+\|u\|_{1}+\|p\|\right) .
$$

Now we take $k \in\left[k_{1}, k_{2}\right]$, where $k_{1}$ and $k_{2}$ are defined as (1.11). Then $\{u, p+k\}$ also solves (Pr. LF). By the definition,

$$
\left|k_{1}\right|,\left|k_{2}\right| \leq\left|\sigma_{n}(u, p)\right|+|g| \leq 2 g \text { on } \Gamma .
$$

This implies $|k| \leq 2|\Gamma|^{-1 / 2}\|g\|_{0, \Gamma}$. As a result, we have

$$
\begin{aligned}
\|u\|_{2}+\|p+k\|_{1} & \leq\|u\|_{2}+\|p\|_{1}+|k| \sqrt{\mid \overline{|\Omega|}} \\
& \leq C\left(\|f\|+\|g\|_{1 / 2, \Gamma}+\|u\|_{1}+\|p\|\right),
\end{aligned}
$$

which completes the proof.

\section{§5. Slip Problem of Friction Type}

In this section, we consider (Pr. SF), which is composed of (1.1), (1.2) with (1.4), and give the proof of Theorem 1.2. The strategy is the same as the previous sections.

We begin with a weak formulation using a variational inequality.

(Pr. WSF). Find $u \in K_{n}$ and $p \in L^{2}(\Omega)$ satisfying

$$
\begin{array}{ll}
a_{\lambda}(u, v-u)+b(v-u, p) & \\
+j\left(v_{\tau}\right)-j\left(u_{\tau}\right) \geq(f, v-u) & \left(\forall v \in K_{n}\right), \\
b(u, \chi)=0 & \left(\forall \chi \in L^{2}(\Omega)\right),
\end{array}
$$


where $j$ denotes

$$
j(\eta)=\int_{\Gamma} g|\eta| d s \quad\left(\eta \in H^{1 / 2}(\Gamma)^{N}\right) .
$$

Fujita [9] proved the following

Proposition 5.1. Assume (3.4), and $g>0$ a.e.in $\Gamma$. Then there is a solution $\{u, p\}$ of (Pr. WSF). Moreover, $u$ is unique; and $p$ is also unique except for an additive constant. In particular, $p$ such that $(p, 1)=0$ is unique.

Let $\varepsilon>0$. We introduce

$$
j_{\varepsilon}(\eta)=\int_{\Gamma} g \rho_{\varepsilon}(\eta) d s \quad\left(\eta \in H^{1 / 2}(\Gamma)^{N}\right),
$$

where $\rho_{\varepsilon}$ denotes the Yosida regularization of $\rho=|z|=\sqrt{z_{1}^{2}+\cdots+z_{N}^{2}}$ for $z \in \mathbb{R}^{N}$ :

$$
\rho_{\varepsilon}(z)= \begin{cases}|z|-\varepsilon / 2 & (|z|>\varepsilon) \\ |z|^{2} /(2 \varepsilon) & (|z| \leq \varepsilon) .\end{cases}
$$

We have

$$
\begin{array}{ll}
\left|j_{\varepsilon}(\eta)-j(\eta)\right| \leq \frac{\varepsilon}{2}\|g\|_{L^{1}(\Omega)} & \left(\eta \in H^{1 / 2}(\Gamma)^{N}\right), \\
\lim _{h \rightarrow 0} \frac{1}{h}\left[j_{\varepsilon}(\eta+h \xi)-j_{\varepsilon}(\eta)\right]=\int_{\Gamma} g \alpha_{\varepsilon}(\eta) \cdot \xi d s & \left(\eta, \xi \in H^{1 / 2}(\Gamma)^{N}\right),
\end{array}
$$

where

$$
\alpha_{\varepsilon}(z)= \begin{cases}z /|z| & (|z|>\varepsilon) \\ z / \varepsilon & (|z| \leq \varepsilon) .\end{cases}
$$

A regularized problem to (Pr. WLF) is

$\left(\operatorname{Pr} . \mathrm{WSF}_{\varepsilon}\right)$. Find $u_{\varepsilon} \in K_{n}$ and $p_{\varepsilon} \in L^{2}(\Omega)$ satisfying

$$
\begin{aligned}
& a\left(u_{\varepsilon}, v-u_{\varepsilon}\right)+b\left(v-u_{\varepsilon}, p_{\varepsilon}\right) \\
& +j_{\varepsilon}\left(v_{\varepsilon, t}\right)-j_{\varepsilon}\left(u_{\varepsilon, t}\right) \geq\left(f, v-u_{\varepsilon}\right) \quad\left(\forall v \in K_{n}\right), \\
& b\left(u_{\varepsilon}, \chi\right)=0 \\
& \left(\forall \chi \in L^{2}(\Omega)\right) \text {. }
\end{aligned}
$$

As Theorem 1.1 is so, Theorem 1.2 is a consequence of the following lemmas; Since the proof of Theorem 1.2 is almost same as that of Theorem 1.1, we skip it. 
Lemma 5.1. Let $\lambda \geq 0$ and $\varepsilon>0$. Suppose that $\Gamma_{D} \neq \emptyset$ if $\lambda=0$. Assume that (3.4) and $g \geq 0$ a.e.in $\Gamma$. Then $\left(\operatorname{Pr} . \mathrm{WSF}_{\varepsilon}\right)$ admits a unique solution $u_{\varepsilon} \in K_{n}$ and $p_{\varepsilon} \in L_{0}^{2}(\Omega)$ characterized by

$$
a\left(u_{\varepsilon}, \varphi\right)+b\left(\varphi, p_{\varepsilon}\right)+\int_{\Gamma} g \alpha_{\varepsilon}\left(u_{\varepsilon, \tau}\right) \cdot \varphi_{\tau} d s=(f, \varphi) \quad\left(\forall \varphi \in K_{n}\right) .
$$

Moreover we have

$$
\left\|u_{\varepsilon}\right\|_{1}+\left\|p_{\varepsilon}\right\| \leq C(\lambda)\left(\|f\|+\|g\|_{\Gamma}\right) .
$$

Lemma 5.2. Let $\lambda \geq 0$. Suppose (1.6), (1.7), (1.9), and (1.10). Let $\varepsilon>0$ and let $\left\{u_{\varepsilon}, p_{\varepsilon}\right\} \in K_{n} \times L_{0}^{2}(\Omega)$ solve

$$
\begin{array}{ll}
a_{\lambda}\left(u_{\varepsilon}, \varphi\right)+b\left(\varphi, p_{\varepsilon}\right)+\int_{\Gamma} g \alpha_{\varepsilon}\left(u_{\varepsilon, \tau}\right) \cdot \varphi_{\tau} d s=(f, \varphi) & \left(\forall \varphi \in K_{n}\right), \\
b\left(u_{\varepsilon}, \chi\right)=0 & \left(\forall \chi \in L^{2}(\Omega)\right) .
\end{array}
$$

Then $u_{\varepsilon} \in H^{2}(\Omega)^{N}, p_{\varepsilon} \in H^{1}(\Omega)$ and they satisfy

$$
\begin{array}{ll}
\lambda u_{\varepsilon}-\Delta u_{\varepsilon}+\nabla p_{\varepsilon}=f, \quad \operatorname{div} u_{\varepsilon}=0 & \text { in } \Omega, \\
u_{\varepsilon}=0, & \text { on } \Gamma_{D}, \\
u_{\varepsilon, n}=0, \quad-\sigma_{\tau}\left(u_{\varepsilon}, p_{\varepsilon}\right)=g \alpha_{\varepsilon}\left(u_{\varepsilon, \tau}\right) & \text { on } \Gamma .
\end{array}
$$

Moreover there is a constant $C>0$ independent of $\varepsilon$ and $\lambda$ such that

$$
\left\|u_{\varepsilon}\right\|_{2}+\left\|p_{\varepsilon}\right\|_{1} \leq C\left(\|f\|+\|g\|_{1 / 2, \Gamma}+\left\|u_{\varepsilon}\right\|_{1}+\left\|p_{\varepsilon}\right\|\right) .
$$

We can show Lemma 5.1 in the similar line as that of Lemma 3.1 by making use of Lemma 2.3. We only state

Sketch of the proof of Lemma 5.2. We follow the notation of the proof of Lemma 4.1. We investigate the regularity near $\Gamma$ and only state the case of $N=3$. Suppose that we have reached

$$
\begin{aligned}
& \tilde{a}_{\lambda}(\tilde{u}, \tilde{\varphi})+\tilde{b}(\tilde{\varphi}, \tilde{p})+\int_{S} \tilde{g} \alpha\left(\tilde{u}_{\tau}\right) \cdot \tilde{\varphi}_{\tau} d y^{\prime} \\
& =\int_{Q} \tilde{f} \cdot \tilde{\varphi}|\operatorname{Jac} \Phi| d y+\tilde{F}(\tilde{\varphi}), \quad\left(\forall \tilde{\varphi} \in K_{\tau}(Q)\right), \\
& \tilde{b}(\tilde{u}, \tilde{\chi})=\tilde{G}(\tilde{\chi}), \\
& \left(\forall \tilde{\chi} \in L^{2}(Q)\right),
\end{aligned}
$$


where $\tilde{u}_{\tau}=\left(\tilde{u}_{1}, \tilde{u}_{2}, 0\right), \tilde{\varphi}_{\tau}=\left(\tilde{\varphi}_{1}, \tilde{\varphi}_{2}, 0\right)$, and

$$
K_{\tau}(Q)=\left\{\varphi \in K(Q) \mid \varphi_{3}=0 \text { on } S\right\} .
$$

Put $\hat{u}=\left(\tilde{u}_{1}, 0,0\right)$. Taking

$$
\tilde{\varphi}=v \equiv D_{-h}\left(D_{h} \hat{u}\right) \in K_{n}\left(Q_{1}\right),
$$

we obtain by $(4.17)-(4.20)$

$$
\left\|\nabla_{y}\left(D_{h} \hat{u}\right)\right\|_{Q} \leq C_{3}\left(\|\tilde{u}\|_{1, Q}+\|\tilde{p}\|_{Q}+\|\tilde{g}\|_{00, S}+\|\tilde{f}\|_{Q}\right),
$$

in other words

$$
\left\|\nabla_{y}\left(D_{h} \tilde{u}_{1}\right)\right\|_{Q} \leq C_{3}\left(\|\tilde{u}\|_{1, Q}+\|\tilde{p}\|_{Q}+\|\tilde{g}\|_{00, S}+\|\tilde{f}\|_{Q}\right) .
$$

(Here $\nabla_{y}\left(D_{h} \hat{u}\right)$ denotes the tensor product, whereas $\nabla_{y}\left(D_{h} \tilde{u}_{1}\right)$ denotes the usual gradient.) There we note that $\alpha$ which appeared in (4.19) should be replaced by $\alpha_{1}$, where $\alpha=\left(\alpha_{1}, \alpha_{2}, \alpha_{3}\right)$. In the same way, we have

$$
\left\|\nabla_{y}\left(D_{h} \tilde{u}_{2}\right)\right\|_{Q} \leq C_{3}\left(\|\tilde{u}\|_{1, Q}+\|\tilde{p}\|_{Q}+\|\tilde{g}\|_{00, S}+\|\tilde{f}\|_{Q}\right)
$$

by choosing $\hat{u}=\left(0, \tilde{u}_{2}, 0\right)$. These lead to

$$
\left\|\nabla_{y}\left(D_{h} \tilde{u}\right)\right\|_{Q} \leq C_{3}\left(\|\tilde{u}\|_{1, Q}+\|\tilde{p}\|_{Q}+\|\tilde{g}\|_{00, S}+\|\tilde{f}\|_{Q}\right)
$$

and the rest is the same as the proof of Lemma 4.1 .

\section{§6. Remarks on Regularity Results for Stokes Problems}

This section is devoted to the boundary value problems of Neumann type composed of

$$
\begin{cases}\lambda u-\Delta u+\nabla p=f, & \operatorname{div} u=0 \\ u=0 & \text { in } \Omega, \\ \text { on } \Gamma_{D}\end{cases}
$$

with one of the following conditions:

$$
\begin{array}{lll}
u_{\tau}=0, \quad \sigma_{n}=\omega_{n} & \text { on } \Gamma ; \\
u_{n}=0, \quad \sigma_{\tau}=\omega_{\tau} & \text { on } \Gamma ; \\
\sigma=\omega & & \text { on } \Gamma
\end{array}
$$


where $\omega_{n}, \omega_{\tau}$ and $\omega$ are given functions defined on $\Gamma$. If a smooth vector field $u$ and a smooth scalar filed $p$ satisfy (6.1) and (6.2), then by (2.8)

$$
a_{\lambda}(u, \varphi)+b(\varphi, p)=\int_{\Gamma} \omega_{n} \varphi_{n} d s+(f, \varphi) \quad\left(\forall \varphi \in K_{\tau}\right) .
$$

From this observation, $\{u, p\} \in \hat{K}_{\tau} \times L^{2}(\Omega)$ satisfying this equation may be regarded as a weak solution of (6.1) with (6.2). Furthermore we have

Theorem 6.1. Let $\lambda \geq 0$ and $0 \leq k \in \mathbb{Z}$. Suppose that

$$
\begin{array}{ll}
\overline{\Gamma_{D}} \cap \bar{\Gamma}=\emptyset, & \Gamma_{D}, \Gamma \text { are of class } C^{k+2}, C^{k+3} ; \\
f \in H^{k}(\Omega)^{N} . &
\end{array}
$$

Let $\omega_{n} \in H^{k+1 / 2}(\Gamma)$. Moreover, suppose that $\{u, p\} \in K_{\tau} \times L^{2}(\Omega)$ is a solution of

$$
\begin{array}{ll}
a_{\lambda}(u, \varphi)+b(\varphi, p)=\int_{\Gamma} \omega_{n} \varphi_{n} d s+(f, \varphi), & \left(\forall \varphi \in K_{\tau}\right), \\
b(u, \chi)=0 & \left(\forall \chi \in L^{2}(\Omega)\right) .
\end{array}
$$

Then $u \in H^{k+2}(\Omega)^{N}, p \in H^{k+1}(\Omega)$, and they satisfy

$$
\begin{array}{ll}
\lambda u-\Delta u+\nabla p=f, \quad \operatorname{div} u=0 & \text { in } \Omega, \\
u=0, & \text { on } \Gamma_{D}, \\
u_{\tau}=0, \quad \sigma_{n}(u, p)=\omega_{n} & \text { on } \Gamma .
\end{array}
$$

Moreover

$$
\|u\|_{k+2}+\|p\|_{k+1} \leq C(\lambda, k)\left(\|f\|_{k}+\left\|\omega_{n}\right\|_{k+1 / 2, \Gamma}\right) .
$$

Remark. In the above theorem, we have assumed the existence of $\{u, p\}$ satisfying (6.7) and (6.8). However, if $\Gamma_{D} \neq \emptyset$, such $\{u, p\}$ always exists under the condition that $f \in L^{2}(\Omega)$ and $\omega_{n} \in L^{2}(\Gamma)$. Actually, a linear functional on $K_{\tau}$ defined as the right-hand side of $(6.7)$ is bounded and its bound is given by $C\left(\|f\|+\left\|\omega_{n}\right\|_{0, \Gamma}\right)$. The bilinear form $a$ is coercive in $K_{\tau} \times K_{\tau}$ as was stated before, and moreover the bilinear form $b$ satisfies the inf-sup condition (2.11). Therefore a well-known result on abstract variational problem with constraints (Theorem 5.6 of [13], Corollary I-4.1 of [17]) is applicable. Hence 
we immediately obtain a unique existence of $\{u, p\} \in K_{\tau} \times L^{2}(\Omega)$ of a solution of $(6.7),(6.8)$ with

$$
\|u\|_{1}+\|p\| \leq C\left(\|f\|+\left\|\omega_{n}\right\|_{0, \Gamma}\right) .
$$

Of course, it can be verified in the same manner as the proof of Lemma 3.2 with the aid of Lemma 2.1. In the case of $\Gamma_{D}=\emptyset$, an additional assumption $\lambda>0$ is required.

Theorem 6.2. Let $\lambda \geq 0$ and $0 \leq k \in \mathbb{Z}$. Suppose that (6.5) and (6.6) hold. Let $\omega_{\tau} \in H^{k+1 / 2}(\Gamma)^{N}$ and let $\{u, p\} \in K_{n} \times L_{0}^{2}(\Omega)$ be a solution of

$$
\begin{aligned}
& a_{\lambda}(u, \varphi)+b(\varphi, p)=\int_{\Gamma} \omega_{\tau} \cdot \varphi_{\tau} d s+(f, \varphi), \quad\left(\forall \varphi \in K_{n}\right), \\
& b(u, \chi)=0 \quad\left(\forall \chi \in L^{2}(\Omega)\right) .
\end{aligned}
$$

Then $u \in H^{k+2}(\Omega)^{N}, p \in H^{k+1}(\Omega)$, and they satisfy

$$
\begin{array}{ll}
\lambda u-\Delta u+\nabla p=f, \quad \operatorname{div} u=0 & \text { in } \Omega, \\
u=0, & \text { on } \Gamma_{D}, \\
u_{n}=0, \quad \sigma_{\tau}(u, p)=\omega_{\tau} & \text { on } \Gamma .
\end{array}
$$

Moreover

$$
\|u\|_{k+2}+\|p\|_{k+1} \leq C(\lambda, k)\left(\|f\|_{k}+\left\|\omega_{\tau}\right\|_{k+1 / 2, \Gamma}\right) .
$$

Theorem 6.3. Let $\lambda \geq 0$ and $0 \leq k \in \mathbb{Z}$. Suppose that (6.5) and (6.6) hold. Let $\omega \in H^{k+1 / 2}(\Gamma)^{N}$ and let $\{u, p\} \in K \times L^{2}(\Omega)$ be a solution of

$$
\begin{aligned}
& a_{\lambda}(u, \varphi)+b(\varphi, p)=\int_{\Gamma} \omega \cdot \varphi d s+(f, \varphi), \quad(\forall \varphi \in K), \\
& b(u, \chi)=0 \quad\left(\forall \chi \in L^{2}(\Omega)\right) .
\end{aligned}
$$

Then $u \in H^{k+2}(\Omega)^{N}, p \in H^{k+1}(\Omega)$, and they satisfy

$$
\begin{array}{ll}
\lambda u-\Delta u+\nabla p=f, \quad \operatorname{div} u=0 & \text { in } \Omega, \\
u=0, & \text { on } \Gamma_{D}, \\
\sigma(u, p)=\omega & \text { on } \Gamma .
\end{array}
$$

Moreover

$$
\|u\|_{k+2}+\|p\|_{k+1} \leq C(\lambda, k)\left(\|f\|_{k}+\|\omega\|_{k+1 / 2, \Gamma}\right) .
$$


Since proofs of these three theorems are done in the essentially same way, we prove the first one.

Proof of Theorem 6.1. We first prove the case of $k=0$. As is stated before, it suffices to investigate the regularity near $\Gamma$. Let $x_{0} \in \Gamma$ and $U_{0} \subset \mathbb{R}^{N}$ be a neighbourhood of $x_{0}$. Again we take $R>0, U \subset U_{0}$ and a one-to-one mapping $\Phi=\left(\Phi_{1}, \ldots, \Phi_{N}\right)$ from $U$ onto $\tilde{U} \subset \mathbb{R}_{y}^{N}$ described in the proof of Lemma 4.1. (We follow the notation of the proof of Lemma 4.1.) However in this case we assume that $\Phi$ is only of class $C^{2}$.

We set $y=\Phi(x)=\left(\Phi_{1}(x), \ldots, \Phi_{N}(x)\right)$,

$$
\tilde{u}(y)=\left(\theta^{2} u\right)(x), \quad \tilde{p}(y)=\left(\theta^{2} p\right)(x)
$$

and

$$
\tilde{f}(y)=\left(\theta^{2} f\right)(x), \quad \tilde{\omega}(y)=\left(\theta^{2} \omega_{n}\right)(x),
$$

where $\theta \in C^{\infty}\left(\mathbb{R}^{N}\right)$ is a cut-off function subject to (3.3). Then we deduce

$$
\begin{aligned}
& \tilde{a}_{\lambda}(\tilde{u}, \tilde{\varphi})+\tilde{b}(\tilde{\varphi}, \tilde{p})=-\int_{S_{R}} \tilde{\omega} \tilde{\varphi_{N}} d y^{\prime} \\
& +\int_{Q_{R}} \tilde{f} \cdot \tilde{\varphi}|\operatorname{Jac} \Phi| d y+\tilde{F}(\tilde{\varphi}), \quad\left(\forall \tilde{\varphi} \in K_{\tau}\left(Q_{R}\right)\right), \\
& \text { (6.13) } \tilde{b}(\tilde{u}, \tilde{\chi})=\tilde{G}(\tilde{\chi}) \text {, } \\
& \left(\forall \tilde{\chi} \in L^{2}\left(Q_{R}\right)\right) .
\end{aligned}
$$

If we proceed in the same way as in the proof of Lemma 4.1, we should assume that $\Phi$ is a $C^{3}$ mapping. However we can avoid such issue by employing a trick of Bello [2].

Let $R_{1} \in(0, R)$. We set $\varepsilon=R-R_{1}, R_{2}=R-\varepsilon / 2, R_{3}=R-\varepsilon / 4$ and simply write $Q_{i}=Q_{R_{i}}, S_{i}=S_{R_{i}}$ for $i=0,1,2,3$. ( $R_{0}$ is understood as $R$.) We shall not distinguish $v \in K(Q)$ with their restrictions into $Q_{i}(i=1,2,3)$; $v \in K\left(Q_{i}\right)$.

We may assume that

$$
\tilde{u} \in K_{\tau}\left(Q_{1}\right), \quad \tilde{\omega} \in H_{00}^{1 / 2}\left(S_{1}\right) .
$$

Let $0<h<\varepsilon / 4$. We fix $i \in\{1,2, \ldots, N-1\}$ and write $D_{ \pm h}=D_{ \pm h}^{i}$ for simplicity. Suppose $\tilde{\varphi} \in K_{\tau}\left(Q_{2}\right), \tilde{\chi} \in L^{2}\left(Q_{2}\right)$ and substitute $D_{-h} \tilde{\varphi}, D_{-h} \tilde{\chi}$ into (6.12), (6.13) as test functions. Then

$$
\begin{array}{ll}
\tilde{a}_{\lambda}\left(D_{h} \tilde{u}, \tilde{\varphi}\right)+\tilde{b}\left(\tilde{\varphi}, D_{h} \tilde{p}\right)=\tilde{F}_{0}(\tilde{\varphi}), & \left(\forall \tilde{\varphi} \in K_{\tau}\left(Q_{2}\right)\right), \\
\tilde{b}\left(D_{h} \tilde{u}, \tilde{\chi}\right)=\tilde{G}_{0}(\tilde{\chi}), & \left(\forall \tilde{\chi} \in L^{2}\left(Q_{2}\right)\right),
\end{array}
$$


where

$$
\begin{gathered}
\tilde{F}_{0}(\tilde{\varphi})=-\int_{S_{R}} \tilde{\omega} D_{-h} \tilde{\varphi}_{N} d y^{\prime}+\int_{Q_{R}} \tilde{f} \cdot\left(D_{-h} \tilde{\varphi}\right)|\operatorname{Jac} \Phi| d y+\tilde{F}\left(D_{-h} \tilde{\varphi}\right) \\
-\int_{Q_{R}}\left(s_{h} \tilde{e}_{i j}(\tilde{u})\right)\left[\left(D_{h} \frac{\partial \Phi_{l}}{\partial x_{j}}\right) \frac{\partial \tilde{\varphi}_{i}}{\partial y_{l}}+\left(D_{h} \frac{\partial \Phi_{l}}{\partial x_{i}}\right) \frac{\partial \tilde{\varphi}_{j}}{\partial y_{l}}\right] d y \\
\tilde{G}_{0}(\tilde{\chi})=\tilde{G}\left(D_{-h} \chi\right)+\int_{Q_{R}}\left(s_{h} \tilde{\chi}\right) D_{h}\left(\frac{\partial \Phi_{k}}{\partial y_{i}}|\operatorname{Jac} \Phi|\right) \frac{\partial \varphi_{i}}{\partial y_{k}} d y .
\end{gathered}
$$

We claim:

$$
\begin{array}{ll}
\left|\tilde{a}_{\lambda}(v, w)\right| \leq C(\lambda)\|v\|_{1, Q_{2}}\|w\|_{1, Q_{2}} & \left(\forall v, w \in K_{\tau}\left(Q_{2}\right)\right) ; \\
\tilde{a}_{\lambda}(v, v) \mid \geq C\|v\|_{1, Q_{2}}^{2} & \left(\forall v \in K_{\tau}\left(Q_{2}\right)\right) ; \\
C\|\chi\|_{Q_{2}} \leq \sup _{v \in K_{\tau}\left(Q_{2}\right)} \frac{\tilde{b}(v, \chi)}{\|v\|_{1, Q_{2}}} & \left(\forall \chi \in L^{2}(\Omega)\right) ; \\
\left|\tilde{F}_{0}(\varphi)\right| \leq C\left(\|\tilde{u}\|_{1, Q_{2}}+\|\tilde{p}\|_{Q_{2}}\right. & \\
\left.+\|\tilde{f}\|_{Q_{3}}+\|\tilde{\omega}\|_{00, S_{1}}\right)\|\varphi\|_{1, Q_{2}} & \left(\forall \varphi \in K_{\tau}\left(Q_{2}\right)\right) ; \\
\left|\tilde{G}_{0}(\chi)\right| \leq C\|\tilde{u}\|_{1, Q_{2}}\|\chi\|_{Q_{2}} & \left(\forall \chi \in L^{2}(\Omega)\right) .
\end{array}
$$

(6.16) is obvious. (6.17) is a consequence of (4.21). To see (6.18), let $v \in$ $K_{\tau}\left(Q_{2}\right), \chi \in L^{2}\left(Q_{2}\right)$, and set

$$
\hat{v}(x)=\left\{\begin{array}{ll}
v(y) & y \in Q_{2} \\
0 & \text { otherwise, }
\end{array} \quad \hat{\chi}(x)= \begin{cases}\chi(y) & y \in Q_{2} \\
0 & \text { otherwise. }\end{cases}\right.
$$

Since $\hat{v} \in K_{\tau}, \hat{\chi} \in L^{2}(\Omega)$, by $(2.11)$, we have

$$
\beta\|\chi\|_{Q_{2}}=\beta\|\hat{\chi}\| \leq \frac{|b(\hat{v}, \hat{\chi})|}{\|\hat{v}\|_{1}} \leq C \frac{|\tilde{b}(v, \chi)|}{\|v\|_{1, Q_{2}}} .
$$

Finally (6.19) and (6.20) are easily obtained in the standard manner.

Now, (6.16)-(6.20) enable us to apply to (6.14)(6.15) the theorem on abstract variational problem with constrains; Theorem 5.6 of [13] or Corollary I-4.1 of [17]. As a result, $D_{h} \tilde{u} \in K_{\tau}\left(Q_{2}\right), D_{h} \tilde{p} \in L^{2}\left(Q_{2}\right)$ and

$$
\left\|D_{h} \tilde{u}\right\|_{1, Q_{2}}+\left\|D_{h} \tilde{p}\right\|_{Q_{2}} \leq C\left(\|\tilde{u}\|_{1, Q_{2}}+\|\tilde{p}\|_{Q_{2}}+\|\tilde{f}\|_{Q_{3}}+\|\tilde{\omega}\|_{00, S_{1}}\right) .
$$

Letting $h \downarrow 0$, we obtain

$$
\left\|\frac{\partial \tilde{u}}{\partial y_{j}}\right\|_{1, Q_{2}}+\left\|\frac{\partial \tilde{p}}{\partial y_{j}}\right\|_{Q_{2}} \leq C\left(\|\tilde{u}\|_{1, Q_{2}}+\|\tilde{p}\|_{Q_{2}}+\|\tilde{f}\|_{Q_{3}}+\|\tilde{\omega}\|_{00, S_{1}}\right)
$$


for $j=1, \ldots, N-1$, and hence

$$
\sum_{j=1}^{N-1}\left\|\zeta_{j}\right\|_{1, Q_{2}} \leq C\left(\|\tilde{u}\|_{1, Q_{2}}+\|\tilde{p}\|_{Q_{2}}+\|\tilde{f}\|_{Q_{3}}+\|\tilde{\omega}\|_{00, S_{1}}\right)
$$

where $\zeta_{j}=\partial \tilde{u} / \partial y_{j}$. This means that $\eta=\left.\tilde{u}_{N}\right|_{S_{2}} \in H^{3 / 2}\left(S_{2}\right)$ and

$$
\|\eta\|_{3 / 2, S_{2}} \leq C\left(\|\tilde{u}\|_{1, Q_{2}}+\|\tilde{p}\|_{Q_{2}}+\|\tilde{f}\|_{Q_{3}}+\|\tilde{\omega}\|_{00, S_{1}}\right) .
$$

Summing up the above estimates, by the argument of the partition of unity, we have

$$
\|\beta\|_{3 / 2, \Gamma} \leq C\left(\|u\|_{1, \Omega}+\|p\|_{\Omega}+\left\|\omega_{n}\right\|_{1 / 2, \Gamma}+\|f\|_{\Omega}\right)
$$

where $\beta=\left.u_{n}\right|_{\Gamma}$. Therefore, by Cattabriga's result, we get the desired result for $k=0$.

We proceed to the general case $k \geq 0$. Let $\alpha=\left(\alpha_{1}, \ldots, \alpha_{N-1}\right)$ be a multi-index with $|\alpha|=k$ and set

$$
\partial^{\alpha}=\left(\frac{\partial}{\partial y_{1}}\right)^{\alpha_{1}} \cdots\left(\frac{\partial}{\partial y_{N-1}}\right)^{\alpha_{N-1}}
$$

Suppose that we have obtained the desired result for $k \geq 0$. We may assume

$$
\partial^{\alpha} \tilde{u} \in K_{\tau}\left(Q_{1}\right), \quad \partial^{\alpha} \tilde{\omega} \in H_{00}^{1 / 2}\left(S_{1}\right),
$$

then we have

$$
\begin{array}{ll}
\tilde{a}_{\lambda}\left(D_{h}\left(\partial^{\alpha} \tilde{u}\right), \tilde{\varphi}\right)+\tilde{b}\left(\tilde{\varphi}, D_{h}\left(\partial^{\alpha} \tilde{p}\right)\right)=\tilde{F}_{k}(\tilde{\varphi}), & \left(\forall \tilde{\varphi} \in K_{\tau}\left(Q_{2}\right)\right), \\
\tilde{b}\left(D_{h}\left(\partial^{\alpha} \tilde{u}\right), \tilde{\chi}\right)=\tilde{G}_{k}(\hat{\chi}), & \left(\forall \tilde{\chi} \in L^{2}\left(Q_{2}\right)\right),
\end{array}
$$

where $\tilde{F}_{k}$ and $\tilde{G}_{k}$ are functionals such as

$$
\begin{array}{rlrl}
\left|\tilde{F}_{k}(\varphi)\right| \leq C\left(\|\tilde{u}\|_{k+1, Q_{2}}+\|\tilde{p}\|_{k, Q_{2}}\right. & \\
\left.+\|\tilde{f}\|_{k, Q_{3}}+\left\|\partial^{\alpha} \tilde{\omega}\right\|_{00, S_{1}}\right)\|\varphi\|_{1, Q_{2}} & & \left(\forall \varphi \in K_{\tau}\left(Q_{2}\right)\right), \\
\left|\tilde{G}_{k}(\chi)\right| \leq C\|\tilde{u}\|_{k+1, Q_{2}}\|\chi\|_{Q_{2}} & & \left(\forall \chi \in L^{2}(\Omega)\right) .
\end{array}
$$

In fact, we firstly assume that $\tilde{\varphi} \in K_{\tau}\left(Q_{2}\right), \tilde{\chi} \in L^{2}\left(Q_{2}\right)$ are sufficiently smooth and then substitute $(-1)^{|\alpha|} \partial^{\alpha} D_{-h} \hat{\varphi},(-1)^{|\alpha|} \partial^{\alpha} D_{-h} \hat{\chi}$ into (6.12), (6.13) as new $\tilde{\varphi}, \tilde{\chi}$. Then, by virtue of integration by parts and the density argument, we 
have (6.22) and (6.23). Again repeating the argument for $k=0$, we obtain $D_{h}\left(\partial^{\alpha} \tilde{u}\right) \in K_{\tau}\left(Q_{2}\right), D_{h}\left(\partial^{\alpha} \tilde{p}\right) \in L^{2}\left(Q_{2}\right)$ and

$$
\begin{aligned}
& \left\|D_{h}\left(\partial^{\alpha} \tilde{u}\right)\right\|_{1, Q_{2}}+\left\|D_{h}\left(\partial^{\alpha} \tilde{p}\right)\right\|_{Q_{2}} \\
& \quad \leq C\left(\|\tilde{u}\|_{k+1, Q_{2}}+\|\tilde{p}\|_{k, Q_{2}}+\|\tilde{f}\|_{k, Q_{3}}+\left\|\partial^{\alpha} \tilde{\omega}\right\|_{00, S_{1}}\right) .
\end{aligned}
$$

Therefore, as before, we deduce the desired result for $k+1$. The proof is completed by the induction.

\section{$\S 7 . \quad$ Concluding Remarks}

(A) Convergence rate. The following theorem provides how fast $\left\{u_{\varepsilon}, p_{\varepsilon}\right\}$ converges to $\{u, p\}$, where $\left\{u_{\varepsilon}, p_{\varepsilon}\right\}$ and $\{u, p\}$ are solutions of $\left(\operatorname{Pr} . \mathrm{WLF}_{\varepsilon}\right)$ and (Pr. WLF), respectively. This result gives useful information in the numerical approximation of these problems.

Theorem 7.1. Let $\lambda \geq 0$ and $\varepsilon>0$. Suppose $\Gamma_{D} \neq \emptyset$ if $\lambda=0$. Assume that (3.4) and $g \geq 0$ a.e. in $\Gamma$. Let $\{u, p\}$ and $\left\{u_{\varepsilon}, p_{\varepsilon}\right\}$ be solutions of $(\operatorname{Pr} . \mathrm{WLF})$ and $\left(\operatorname{Pr} . \mathrm{WLF}_{\varepsilon}\right)$, respectively. Then

$$
\left\|u_{\varepsilon}-u\right\|_{1}+\left\|\tilde{p}_{\varepsilon}-\tilde{p}\right\| \leq C \sqrt{\varepsilon}\|g\|_{L^{1}(\Gamma)},
$$

where $\tilde{p}=p-|\Omega|^{-1}(p, 1), \tilde{p}_{\varepsilon}=p_{\varepsilon}-|\Omega|^{-1}\left(p_{\varepsilon}, 1\right)$ with $|\Omega|$ being the Lebesgue measure of $\Omega$ in $\mathbb{R}^{N}$.

Proof. Substituting $v=u_{\varepsilon}$ and $\varphi=u_{\varepsilon}-u$ into (3.2) and (3.10), respectively, we have

$$
\begin{aligned}
& a_{\lambda}\left(u, u_{\varepsilon}-u\right)+j\left(u_{\varepsilon, n}\right)-j\left(u_{n}\right) \geq\left(f, u_{\varepsilon}-u\right), \\
& a_{\lambda}\left(u_{\varepsilon}, u_{\varepsilon}-u\right)+\int_{\Gamma} g \alpha_{\varepsilon}\left(u_{\varepsilon, n}\right)\left(u_{\varepsilon, n}-u_{n}\right) d s=\left(f, u_{\varepsilon}-u\right) .
\end{aligned}
$$

Hence

$$
a_{\lambda}\left(u_{\varepsilon}-u, u_{\varepsilon}-u\right) \leq j\left(u_{\varepsilon, n}\right)-j\left(u_{n}\right)+\int_{\Gamma} g \alpha_{\varepsilon}\left(u_{\varepsilon, n}\right)\left(u_{n}-u_{\varepsilon, n}\right) d s,
$$

and by (3.5), (3.15), (2.3) (or (2.6))

$$
C\left\|u_{\varepsilon}-u\right\|_{1}^{2} \leq\left|j\left(u_{\varepsilon, n}\right)-j\left(u_{n}\right)\right|+\left|j_{\varepsilon}\left(u_{n}\right)-j_{\varepsilon}\left(u_{\varepsilon, n}\right)\right| \leq \varepsilon\|g\|_{L^{1}(\Gamma)} .
$$

We proceed to the pressure part. Putting $q_{\varepsilon}=\tilde{p}_{\varepsilon}-\tilde{p}$, we have

$$
a_{\lambda}\left(u-u_{\varepsilon}, \varphi\right)=b\left(\varphi, q_{\varepsilon}\right) \quad\left(\forall \varphi \in H_{0}^{1}(\Omega)^{N}\right) .
$$


In fact, for an arbitrary $\varphi \in H_{0}^{1}(\Omega)^{N}$, substituting $v=u \pm \varphi$ into (3.2), we get

$$
a_{\lambda}(u, \varphi)+b(\varphi, p)=(f, \varphi) .
$$

Combining this with $(3.10)$, we deduce $(7.3)$. Since $\left(q_{\varepsilon}, 1\right)=0$, by Lemma 2.4, we can take $\psi_{\varepsilon} \in H_{0}^{1}(\Omega)^{N}$ subject to $\operatorname{div} \psi_{\varepsilon}=q_{\varepsilon}$ in $\Omega$ and $\left\|\psi_{\varepsilon}\right\|_{1} \leq C\left\|q_{\varepsilon}\right\|$. Now substituting into $(7.3) \varphi=\psi_{\varepsilon}$, we obtain

$$
\left\|q_{\varepsilon}\right\|^{2}=a_{\lambda}\left(u-u_{\varepsilon}, \psi_{\varepsilon}\right) \leq C\left\|u-u_{\varepsilon}\right\|_{1}\left\|\psi_{\varepsilon}\right\|_{1} \leq C\left\|u-u_{\varepsilon}\right\|_{1}\left\|q_{\varepsilon}\right\| .
$$

This, together with (7.2), implies (7.1).

Concerning $\left(\mathrm{Pr} . \mathrm{WSF}_{\varepsilon}\right)$ and (Pr. WSF), we also have a corresponding result. The proof is omitted.

Theorem 7.2. Let $\lambda \geq 0$ and $\varepsilon>0$. Suppose that $\Gamma_{D} \neq \emptyset$ if $\lambda=0$. Assume that (3.4) and $g \geq 0$ a.e.in $\Gamma$. Let $\{u, p\}$ and $\left\{u_{\varepsilon}, p_{\varepsilon}\right\}$ be solutions of (Pr. WSF) and $\left(\operatorname{Pr} . \mathrm{WSF}_{\varepsilon}\right)$, respectively. Then

$$
\left\|u_{\varepsilon}-u\right\|_{1}+\left\|\tilde{p}_{\varepsilon}-\tilde{p}\right\| \leq C \sqrt{\varepsilon}\|g\|_{L^{1}(\Gamma)} .
$$

(B) Full stress problem of friction type. We consider the full stress problem of friction type, (Pr. F), which is composed of (1.1), (1.2) and

$$
-\sigma(u, p) \in g \partial|u| \quad \text { on } \Gamma,
$$

where $\partial|\cdot|$ denotes a graph on $\mathbb{R}^{N}$ defined by (1.5) with $m=N$. It is equivalent to

$$
|\sigma| \leq g, \quad \sigma \cdot u+g|u|=0 \quad \text { on } \Gamma .
$$

The existence and uniqueness/non-uniqueness of a weak solution to (Pr. F) are discussed by means of a variational inequality in Fujita and Kawarada [12]. In the previous paper [26], we assumed $g \in H^{1}(\Gamma) \cap L^{\infty}(\Gamma)$ to derive the $H^{2}-H^{1}$ regularity of the weak solution. However, in the same way as that of Theorem 1.2 , we can prove

Theorem 7.3. Let $\lambda \geq 0$. Assume that $\Gamma_{D} \neq \emptyset$ if $\lambda=0$. Suppose that $\bar{\Gamma} \cap \overline{\Gamma_{D}}=\emptyset$ and that $\Gamma_{D}, \Gamma$ are of class $C^{2}, C^{4}$, respectively. Suppose also that $g \in H^{1 / 2}(\Gamma)$ and $g \geq 0$ a.e., and finally let $f \in L^{2}(\Omega)^{N}$. Then there exists a solution $\{u, p\} \in H^{2}(\Omega)^{N} \times H^{1}(\Omega)$ of $(\operatorname{Pr} . \mathrm{F})$. $u$ is unique, while $p$ is 
unique except for an additive constant. The range of the additive constant to $p$ is limited to $\{0\}$ or to a finite closed interval. Furthermore

$$
\|u\|_{2}+\|p\|_{1} \leq C\left(\|f\|+\|g\|_{1 / 2, \Gamma}+\|u\|_{1}+\|p\|\right)
$$

for any solution $\{u, p\}$ of (Pr. F).

Remark. As for the non-uniqueness of $p$, see Remark below Theorem 1.1 or an example in [26].

(C) Regularity for $\left(\mathbf{P r} . \mathbf{W L F}_{\varepsilon}\right)$. Concerning the regularity of a solution $\left\{u_{\varepsilon}, p_{\varepsilon}\right\}$ of $\left(\operatorname{Pr}\right.$. $\left.\mathrm{WLF}_{\varepsilon}\right)$, we can easily derive $u_{\varepsilon} \in H^{2}, p_{\varepsilon} \in H^{1}$ by making use of

Lemma 7.1. Let $\alpha$ be a mapping of $\mathbb{R}^{m} \rightarrow \mathbb{R}^{m}$ with $\alpha(0)=0$. Moreover assume that there are $L, M>0$ such that

$$
\begin{array}{ll}
\left|\alpha(z)-\alpha\left(z^{\prime}\right)\right| \leq L\left|z-z^{\prime}\right| & \left(\forall z, z^{\prime} \in \mathbb{R}^{m}\right), \\
|\alpha(z)| \leq M & \left(\forall z \in \mathbb{R}^{m}\right) .
\end{array}
$$

Let $\eta \in H^{1 / 2}(\Gamma)^{m}$. Then, if $g \in H^{1 / 2}(\Gamma) \cap L^{\infty}(\Gamma)$, we have $g \alpha(\eta) \in H^{1 / 2}(\Gamma)^{m}$ and

$$
\|g \alpha(\eta)\|_{1 / 2, \Gamma} \leq C(M)\|g\|_{1 / 2, \Gamma}+C\left(L, M,\|g\|_{L^{\infty}(\Gamma)}\right)\|\eta\|_{1 / 2, \Gamma}
$$

Proof. Let $\eta \in H^{1 / 2}(\Gamma)^{m}$. The fact that

$$
\alpha(\eta) \in H^{1 / 2}(\Gamma)^{m} \quad \text { and } \quad\|\alpha(\eta)\|_{1 / 2, \Gamma} \leq C(L, M)\|\eta\|_{1 / 2, \Gamma}
$$

is due to H. Brezis. (Lemme I.1 of [4]; The scalar-valued case is explicitly described there, but it is valid for the vector-valued case.)

Next let us denote by $\hat{g} \in H^{1}(\Omega)$ the weak harmonic extension of $g$. It follows from the maximum principle that $\|\hat{g}\|_{\infty} \leq\|g\|_{L^{\infty}(\Gamma)}$. Let $\eta \in H^{1 / 2}(\Gamma)^{m}$. We take the weak harmonic extension $\hat{\alpha} \in H^{1}(\Omega)^{m}$ of $\alpha(\eta)$. That is, we extend an each component of $\alpha(\eta)$ by a harmonic function on $\Omega$. Again by the maximum principle, we have $\|\hat{\alpha}\|_{\infty} \leq\|\alpha(\eta)\|_{L^{\infty}(\Gamma)} \leq M$. Since $\hat{g} \hat{\alpha} \in H^{1}(\Omega)^{m}$, by the trace theorem,

$$
\|g \alpha(\eta)\|_{1 / 2, \Gamma} \leq C\|\hat{g} \hat{\alpha}\|_{1} \leq C M\|\hat{g}\|_{1}+\|g\|_{L^{\infty}(\Gamma)}\|\hat{\alpha}\|_{1} .
$$


This, together with (7.5), implies the conclusion.

Now, let $\lambda \geq 0$ and $\varepsilon>0$. Suppose (1.6), (1.7), (1.9), and (1.10). (We follow the notation of $\S 3$.) Let $\left\{u_{\varepsilon}, p_{\varepsilon}\right\} \in K_{\tau} \times L^{2}(\Omega)$ solve (Pr. WLF F $_{\varepsilon}$. Furthermore we assume $g \in L^{\infty}(\Gamma)$. We notice that

$$
\left|\alpha_{\varepsilon}(z)-\alpha_{\varepsilon}\left(z^{\prime}\right)\right| \leq \frac{1}{\varepsilon}\left|z-z^{\prime}\right|, \quad\left|\alpha_{\varepsilon}(z)\right| \leq 1 \quad\left(z, z^{\prime} \in \mathbb{R}\right) .
$$

Then Lemmas 3.1 and 7.1 (in the case of $m=1$ ) imply that $\sigma_{n}\left(u_{\varepsilon}, p_{\varepsilon}\right)=$ $-g \alpha_{\varepsilon}\left(u_{\varepsilon, n}\right) \in H^{1 / 2}(\Gamma)$. Hence we have $u_{\varepsilon} \in H^{2}(\Omega)^{N}, p_{\varepsilon} \in L^{2}(\Omega)$, and

$$
\begin{aligned}
\left\|u_{\varepsilon}\right\|_{2}+\left\|p_{\varepsilon}\right\| & \leq C\left(\|f\|+\left\|g \alpha_{\varepsilon}\left(u_{\varepsilon, n}\right)\right\|_{1 / 2, \Gamma}\right) \\
& \leq C\left(\|f\|+C\|g\|_{1 / 2, \Gamma}+C\left(\varepsilon,\|g\|_{L^{\infty}(\Gamma)}\right)\left\|u_{\varepsilon, n}\right\|_{1 / 2, \Gamma}\right) \\
& \leq C\left(\|f\|+C\|g\|_{1 / 2, \Gamma}+C\left(\varepsilon,\|g\|_{L^{\infty}(\Gamma)}\right)\left\|u_{\varepsilon}\right\|_{1}\right)
\end{aligned}
$$

by Theorem 6.1. However in order to derive an estimate which is independent of $\varepsilon$ like as (4.7), we need another device described in $\S 4$.

Remark. We meet the same issue when applying a general regularity theory by Agmon, Douglis and Nirenberg [1].

(D) Optimality of Assumption $\boldsymbol{g} \in \boldsymbol{H}^{\mathbf{1 / 2}}(\boldsymbol{\Gamma})$. Theorem 6.1 claims that

$$
g \alpha_{\varepsilon}\left(u_{\varepsilon, n}\right) \in H^{1 / 2}(\Gamma)
$$

is essential to derive the $H^{2}(\Omega)^{N} \times H^{1}(\Omega)$-regularity of a solution $\left\{u_{\varepsilon}, p_{\varepsilon}\right\} \in$ $K_{\tau} \times L^{2}(\Omega)$ to $\left(\operatorname{Pr}\right.$. $\left.\mathrm{WLF}_{\varepsilon}\right)$. However, if $u_{\varepsilon, n} \geq 1 / \varepsilon$ on $\Gamma$, then (7.6) means $g \in H^{1 / 2}(\Gamma)$.

\section{Appendix A. The Space $H_{00}^{1 / 2}$}

In this appendix, we establish the proof of Lemma 4.2 after having prepared some basic properties on the space $H_{00}^{1 / 2}$.

First we recall its definition. Let $\gamma \subset \mathbb{R}_{y}^{N-1}$ be a bounded domain with the Lipschitz boundary $\partial \gamma$. The distance from $y^{\prime} \in \gamma$ to $\partial \gamma$ is denoted by $\delta=\delta\left(y^{\prime}\right)$. Then

$$
H_{00}^{1 / 2}(\gamma)=\left\{\eta \in H^{1 / 2}(\gamma) \mid \delta^{-1 / 2} \eta \in L^{2}(\gamma)\right\}
$$

is a Hilbert space equipped with the norm

$$
\|\eta\|_{00, \gamma}=\|\eta\|_{H_{00}^{1 / 2}(\gamma)}=\left[\|\eta\|_{H^{1 / 2}(\gamma)}^{2}+\int_{\gamma} \frac{\left|\eta\left(y^{\prime}\right)\right|^{2}}{\delta\left(y^{\prime}\right)} d y^{\prime}\right]^{1 / 2}
$$


We consider a finite cylinder $Q=\gamma \times(0, R) \subset \mathbb{R}_{y}^{N-1} \times \mathbb{R}_{y}$ with $R>0$, and set

$$
K(Q)=\left\{v \in H^{1}(Q) \mid v=0 \text { on } \partial Q \backslash \bar{\gamma}\right\} .
$$

If $v \in K(Q)$, then $\eta=\left.v\right|_{\gamma} \in H_{00}^{1 / 2}(\gamma)$ and $\|\eta\|_{00, \gamma} \leq C\|v\|_{H^{1}(Q)}$. Conversely, every $\eta \in H_{00}^{1 / 2}(\gamma)$ admits an extension $v \in K(Q)$ such that $\left.v\right|_{\gamma}=\eta$ and $\|v\|_{H^{1}(Q)} \leq C\|\eta\|_{00, \gamma}$. Moreover the following equivalence holds true

$$
\|\eta\|_{00, \gamma} \sim \inf \left\{\|v\|_{H^{1}(Q)}|v \in K(Q), v|_{\gamma}=\eta\right\} \sim\|\nabla w\|_{L^{2}(Q)},
$$

where $w \in K(Q)$ denotes the weak harmonic extension $\eta$ into $Q ; \Delta w=0$ in $Q$ and $\left.w\right|_{\gamma}=\eta$. See, for more detail, [23] or [25].

Lemma A.1. Let $\gamma, \gamma^{\prime} \subset \mathbb{R}_{y}^{N-1}$ be bounded domains with Lipschitz boundaries, and assume that $\gamma^{\prime} \subset \gamma$ and $\operatorname{dist}\left(\gamma^{\prime}, \gamma\right)>0$. Let $g \in H_{00}^{1 / 2}\left(\gamma^{\prime}\right)$. Then the zero extension $\hat{g}$ of $g$ into $\gamma$ is in $H_{00}^{1 / 2}(\gamma)$. Furthermore we have

$$
\frac{\partial \hat{g}}{\partial y_{i}} \in H^{-1 / 2}(\gamma) \equiv H_{00}^{1 / 2}(\gamma)^{*}, \quad\left\|\frac{\partial \hat{g}}{\partial y_{i}}\right\|_{H^{-1 / 2}(\gamma)} \leq C\left(\gamma, \gamma^{\prime}\right)\|g\|_{00, \gamma^{\prime}}
$$

for $i=1,2, \ldots, N-1$.

Proof. The first assertion is obvious. We give the proof of the second one only when $N=3$. Set $Q^{\prime}=\gamma^{\prime} \times(0, R / 2)$, and take a smooth function $w$ defined on $\overline{Q^{\prime}}$ subject to $w=0$ on $\partial Q^{\prime} \backslash \bar{\gamma}$. Let $\hat{w}$ be the extension of $w$ by 0 into $\bar{Q}$. Then $\partial \hat{w} / \partial y_{j}, j=1,2,3$, are also smooth on $\bar{Q}$ and vanish on $\partial Q \backslash \bar{\gamma}$. We have by the integration by parts

$$
\int_{Q} \operatorname{curl} v \cdot \nabla \hat{w} d y=\int_{\gamma}\left(v_{2} \frac{\partial \hat{w}}{\partial y_{1}}-v_{1} \frac{\partial \hat{w}}{\partial y_{2}}\right) d y^{\prime} \quad\left(\forall v \in K(Q)^{3}\right) .
$$

Taking $\varphi \in K(Q)$ and substituting $v=(0, \varphi, 0)$ into the above, we obtain

$$
\int_{\gamma} \frac{\partial \hat{w}}{\partial y_{1}} \varphi\left(y^{\prime}, 0\right) d y^{\prime}=\int_{Q}\left(\frac{\partial \varphi}{\partial y_{1}} \frac{\partial \hat{w}}{\partial y_{3}}-\frac{\partial \varphi}{\partial y_{3}} \frac{\partial \hat{w}}{\partial y_{1}}\right) d y .
$$

By the density argument, the identity (A.2) is valid for any $w \in K\left(Q^{\prime}\right)=$ $\left\{v \in H^{1}\left(Q^{\prime}\right) \mid v=0\right.$ on $\left.\partial Q^{\prime} \backslash \overline{\gamma^{\prime}}\right\}$. At this stage, we suppose that $w$ is the weak harmonic extension of $g$ into $Q$. The zero extension of $g$ into $\gamma$ is denoted by $\hat{g}$. Moreover, for an arbitrary $\eta \in H_{00}^{1 / 2}(\gamma)$, let $\varphi \in K(Q)$ be the weak harmonic extension into $Q$. Then (A.2) implies

$$
\int_{\gamma} \frac{\partial \hat{g}}{\partial y_{1}} \eta d y^{\prime}=\int_{Q}\left(\frac{\partial \varphi}{\partial y_{1}} \frac{\partial \hat{w}}{\partial y_{3}}-\frac{\partial \varphi}{\partial y_{3}} \frac{\partial \hat{w}}{\partial y_{1}}\right) d y \text {. }
$$


Now we define $\partial \hat{g} / \partial y_{1} \in H^{-1 / 2}(\gamma)$ through this identity and then get

$$
\left|\int_{\gamma} \frac{\partial \hat{g}}{\partial y_{1}} \eta d y^{\prime}\right| \leq\|\nabla w\|_{L^{2}(Q)}\|\nabla \varphi\|_{L^{2}(Q)} \quad\left(\forall \eta \in H_{00}^{1 / 2}(\gamma)\right) .
$$

The case of $i=2$ is done in the similar way.

Finally we state:

Proof of Lemma 4.2. Although the inequality (4.27) is essentially derived by González Burgos [16], we state another (and a somewhat simpler) proof. Taking the harmonic extension $w$ of $g$ into $Q_{R_{1}}$ and using the same symbol $w$ to indicate the zero extension of $w$ into $Q_{R}$. Writing

$$
D_{h}^{i} w(y)=\frac{1}{h} \int_{0}^{h} s_{t}^{i} \frac{\partial w}{\partial y_{i}}(y) d t, \quad\left(s_{t}^{i}: \text { the shift operator defined by }(4.15)\right)
$$

we obtain, for any $\varphi \in K\left(Q_{R_{2}}\right)$,

$$
\begin{aligned}
\int_{S_{R}}\left(D_{h}^{i} g\right) \varphi\left(y^{\prime}, 0\right) d y^{\prime} & =\frac{1}{h} \int_{0}^{h} \int_{S_{R}}\left(s_{t}^{i} \frac{\partial w}{\partial y_{i}}\left(y^{\prime}, 0\right)\right) \varphi\left(y^{\prime}, 0\right) d y^{\prime} d t \\
& =\frac{-1}{h} \int_{0}^{h} \int_{S_{R}}\left(\frac{\partial w}{\partial y_{i}}\left(y^{\prime}, 0\right)\right) s_{-t}^{i} \varphi\left(y^{\prime}, 0\right) d y^{\prime} d t
\end{aligned}
$$

Since $s_{-t}^{i} \varphi\left(y^{\prime}, 0\right) \in H_{00}^{1 / 2}\left(S_{R}\right)$, by Lemma A.1, we have

$$
\begin{aligned}
\int_{S_{R}}\left|\left(D_{h}^{i} g\right) \varphi\left(y^{\prime}, 0\right)\right| d y^{\prime} & \leq \frac{1}{h} \int_{0}^{h} \int_{S_{R}}\left|\frac{\partial g}{\partial y_{i}}\left(y^{\prime}\right)\right| \cdot\left|s_{-t}^{i} \varphi\left(y^{\prime}, 0\right)\right| d y^{\prime} d t \\
& \leq\left(\frac{1}{h} \int_{0}^{h} d t\right)\left\|\frac{\partial g}{\partial y_{i}}\right\|_{H^{-1 / 2}\left(S_{R}\right)}\left\|s_{-t}^{i} \varphi\right\|_{00, S_{R}} \\
& \leq C(R)\|g\|_{00, S_{R_{1}}}\|\nabla \varphi\|_{L^{2}\left(Q_{R}\right)}
\end{aligned}
$$

which completes the proof.

\section{Acknowledgement}

The author wishes to express his gratitude to Professors Haim Brezis and Hiroshi Fujita for their valuable suggestions. Also he would like to express his thanks to the referee who corrected a misunderstanding of the author concerning Korn's inequality in the first manuscript. 


\section{References}

[1] Agmon, S., Douglis, A. and Nirenberg, L., Estimates near the boundary for the solutions of elliptic partial differential equations satisfying general boundary conditions II, Comm. Pure Appl. Math., 17 (1964), 35-92.

[2] Bello, J. A., $L^{r}$ regularity for the Stokes and Navier-Stokes problems, Ann. Mat. Pura Appl. (4), 170 (1996), 187-206.

[3] Brezis, H., Monotonicity methods in Hilbert spaces and some applications to nonlinear partial differential equations, Contributions to Nonlinear Functional Analysis, (Proc. Sympos., Math. Res. Center, Univ. Wisconsin, Madison, Wis., 1971), Academic Press, New York, (1971), 101-156.

[4] _ Problèm unilatéraux, J. Math. Pures et Appl., 51 (1972), 1-168.

[5] - Opérateurs Maximaux Monotones et Semi-groupes de Contractions dans les Espaces de Hilbert, North-Holland Math. Stud., No. 5, Notas de Matemática (50), NorthHolland, Amsterdam, 1973.

[6] _ Analyse Fonctionnelle — Théory et Application, Masson, Paris, 1983.

[7] Cattabriga, L., Su un problema al contorno relativo al sistema di equazioni di Stokes, Rend. Sem. Mat. Univ. Padova, 31 (1961), 1-33.

[8] Duvaut, G. and Lions, J.L., Les Inéquations en Mécanique et en Physique, Dunod, Paris, 1972.

[9] Fujita, H., A mathematical analysis of motions of viscous incompressible fluid under leak or slip boundary conditions, Mathematical Fluid Mechanics and Modeling, Sūrikaisekikenkyūsho Kōkyūroku, $8 \mathbf{8 8}$ (1994), 199-216.

[10] Non-stationary Stokes flows under leak boundary conditions of friction type, $J$. Comput. Math., 19 (2001), 1-8.

[11] Variational inequalities and nonlinear semi-groups applied to certain nonlinear problems for the Stokes equation, Tosio Kato's Method and Principle for Evolution Equations in Mathematical Physics, The Univ. Tokyo Press, (2002), 70-85.

[12] Fujita, H. and Kawarada, H., Variational inequalities for the Stokes equation with boundary conditions of friction type, Recent Developments in Domain Decomposition Methods and Flow Problems, GAKUTO Internat. Ser. Math. Sci. Appl., 11 (1998), 15-33.

[13] Fujita, H., Saito, N. and Suzuki, T., Operator Theory and Numerical Methods, Stud. Math. Appl. 30, North-Holland, Amsterdam, 2001.

[14] Glowinski, R., Numerical Methods for Nonlinear Variational Problems, Springer-Verlag, New York, 1984.

[15] Ghidaglia, J. M., Régularité des solutions de certains problèms aux limites linéaires Liés aux Équations d'Euler, Comm. P. D. E., 9 (1984), 1265-1298.

[16] González Burgos, M., Regularidad $L^{2}$ del problema de Stokes con condiciones de contorno de tipo Neumann, XIV CEDYA/IV Congress of Applied Mathematics, (electronic: http://www-ma1.upc.es/cedya/comunicaciones/gonzal-b.ps), Univ. Barcelona, Barcelona, 1995.

[17] Girault, V. and Raviart, P.-A., Finite Element Methods for Navier-Stokes Equations, Springer, Berlin, 1986.

[18] Heywood, J. G., Rannacher, R. and Turek, S., Artificial boundaries and flux and pressure conditions for the incompressible Navier-Stokes equations, Internat. J. Numer. Methods Fluids, 22 (1996) 325-352.

[19] Kawarada, H., Fujita, H. and Suito, H., Wave breaking phenomenon on the shore and coastal ecosystems, Advances in Numerical Mathematics, GAKUTO Internat. Ser. Math. Sci. Appl., 12 (1999), 65-74.

[20] Kawarada, H. and Suito, H., Numerical simulation of spilled oil drifted on the sloping beach, Computational fluid and solid mechanics, Elsevier, Amsterdam, (2001), 876-878.

[21] Kōmura, Y., Nonlinear semi-groups in Hilbert space, J. Math. Soc. Japan, 19 (1967), 493-507.

[22] Ladyzhenskaya, O. A., The Mathematical Theory of Viscous Incompressible Flow, Gordon and Breach Sci. Publ., London, 1969. 
[23] Lions, J. L. and Magenes, E., Problémes aux Limites non Homogénes et Applications. I, Dunod, Paris, 1968.

[24] Le Roux, C. and Reddy, B. D., The steady Navier-Stokes equations with mixed boundary conditions: application to free boundary flows, Nonlinear Anal., 20 (1993), 1043-1068.

[25] Saito, N. and Fujita, H., Remarks on traces of $H^{1}$-functions defined in a domain with corners, J. Math. Sci. Univ. Tokyo, 7 (2000), 325-345.

[26] Regularity of solutions to the Stokes equations under a certain nonlinear boundary condition, The Navier-Stokes Equations, Lecture Notes in Pure and Appl. Math., 223 (2001), 73-86.

[27] Solonnikov, V. A., The solvability of the second initial boundary-value problem for the linear, time-dependent system of Navier-Stokes equations, J. Soviet Math., 10 (1978), 141-155.

[28] Solonnikov, V. A. and Ščadilov, V. E., On a boundary value problem for a stationary system of Navier-Stokes equations, Proc. Steklov Inst. Math., 125 (1973), 186-199.

[29] Wolka, J., Partial Differential Equations, Cambridge University Press, Cambridge, 1987.

[30] Ciarlet, P. G., Mathematical Elasticity. Vol. I. Three-dimensional Elasticity, NorthHolland, Amsterdam, 1988.

[31] Fujita, H., A coherent analysis of Stokes flows under boundary conditions of friction type, J. Comput. Appl. Math., 149 (2002), 57-69. 